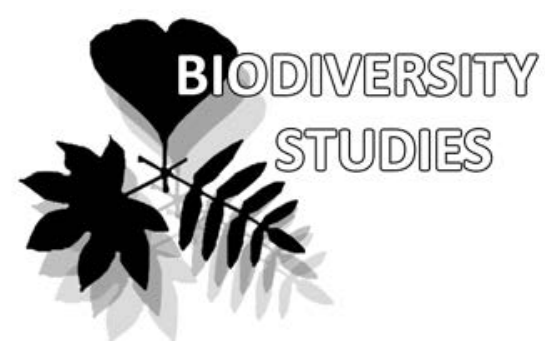

\title{
Liverworts from Attu Island, Near Islands, Aleutian Islands, Alaska (USA) with comparison to the Commander Islands (Russia)
}

\author{
Stephen S. Talbot ${ }^{1 *}$, Wilfred B. Schofield ${ }^{2 \dagger}$, Jiří Váňa ${ }^{3 \dagger}$ \\ $\&$ Sandra L. Talbot ${ }^{4}$
}

Stephen S. Talbot ${ }^{1 *}$

e-mail: stephen_talbot@fws.gov

Wilfred B. Schofield ${ }^{2 \dagger}$

Jiří Váňa ${ }^{3 \dagger}$

Sandra L. Talbot ${ }^{4}$

e-mail: stalbot@usgs.gov

${ }^{1}$ U.S. Fish and Wildlife Service, Anchorage, AK 99503 USA

${ }^{2}$ Department of Botany, University of British Columbia, Vancouver, V6T 1Z4, Canada

${ }^{3}$ Department of Botany, Charles University, 12801 Praha 2, Czech Republic

${ }^{4}$ Alaska Science Center, U.S. Geological Survey, Anchorage, AK 99508 USA

† Deceased

* corresponding authors

Manuscript received: 13.11 .2017

Review completed: 28.06.2018

Accepted for publication: 06.08.2018

Published online: 13.08 .2018

\begin{abstract}
A B S T R A C T
The liverwort flora of Attu Island, the westernmost Aleutian Island in the United States, was studied to assess species diversity in the hyperoceanic sector of the northern boreal subzone. The field study was undertaken in sites selected to represent a spectrum of environmental variation, primarily within the eastern part of the island. Data were analyzed using our own collections on Attu Island, supplemented with information from published reports to compare bryophyte distribution patterns at three levels, the Northern Hemisphere, North America, the Commander Islands of Russia, and Alaska. A total of 112 liverworts were identified and a substantial number, 34 species (30\%), were new reports from Attu Island and one was new to Alaska. Geographic elements dominating the flora included arctomontane $(26 \%)$, arctoboreomontane $(23 \%)$, montane $(20 \%)$, and boreal $(14 \%)$ species, while arctic species were almost absent $(1 \%)$. The liverworts of the Attu Island-Commander Islands region were widespread species with over $70 \%$ circumpolar, or nearly circumpolar; nevertheless large gaps were present in some of their distributions with a floristic depression in liverwort distribution between Attu and the Commander Islands.
\end{abstract}

K e y w o r d s : Hepaticae, phytogeography, northern boreal, hyperoceanic, Bering Land Bridge

\section{P E 3 Ю M E}

Тэлбот С.С., СхофильА В.Б., Ванья И., Тэлбот С.А. Печеночники острова Атту, БАижние острова, Алеуты, Аляска (США) в сравнении с печеночниками Командорских островов (Россия). ПреАставАены результаты исследований флоры печеночников острова Атту, самого западного алеутского острова в США, на основании которых проведена оценка виАового разнообразия печеночников в гиперокеаническом секторе северной подзоны бореальной зоны. Полевые работы проводились на участках, отражающих спектр изменений условий окружающей среды, прежле всего в восточной части острова. В анализ включены наши собственные коллекции с острова Атту и опублиикованные данные Аругих авторов. Проведен анализ распрелеления печеночников на трех уровнях: в Северном полушарии, в Северной Америке, на российских Командорских островах и на А^яске. Было выявлено в общей сложности 112 вилов печеночников, значительная часть которых - 34 вида $(30$ \%) - впервые отмечены на острове Атту, а один виА является новым Аля А^яски. Географический анализ флоры позволиц установить, что в спектре географических элементов преобмадают арктомонтанные (26\%), арктобореомонтанные $(23 \%)$, монтанные $(20 \%)$ и бореальные $(14 \%)$ виды, в то время как арктические виды почти отсутствуют (1\%). Печеночники, совместно встречающиеся на острове Атту и Командорских островах, на 70 \% представлены циркумполярными или почти циркумполярными виАами; тем не менее, существуют большие разАичия межАу флорами печеночников Атту и Командорских островов.

КАючевые слова: Нераticaе, фитогеография, бореальная зона, гиперокеанический кАимат, Берингийский мост суши

Переведено редколегией

\section{N T RODUCTION}

From the northwestern portion of the North America landmass, the Aleutian Island Archipelago extends in an arc $1900 \mathrm{~km}$ into the northern Pacific Ocean from Unimak Island - the easternmost island - through Attu Island, the westernmost island in the United States, toward the Commander Islands of Russia, which comprise the westernmost portion of the archipelago. These windswept, often foggy volcanic islands are of considerable phytogeographic interest because they occur along the southern margin of the Bering Land Bridge and function as an important region for plant dispersal between Asia and North America (Elias \& Crocker 2008). Species dispersal occurs in at least two directions, westward from North America along the Aleutian Archipelago, and eastward from Asia toward mainland 
Alaska and the more temperate North Pacific Coast. Attu Island is of particular interest because of its relatively close proximity (ca. $300 \mathrm{~km}$ ) to the Commander Islands of Russian Aleutian Islands. Hultén (1960) indicated the Aleutian Islands belong to the same floral and vegetational province as the Kamchatka Peninsula of Russia with the strongest vascular plant relationships occurring in the westernmost islands of Attu, Agattu and Alaid. Of the approximately 530 vascular plant species comprising the Aleutian vascular flora, $70 \%$ occur in both Alaska and Kamchatka. Attu Island falls within the boundary of the U.S. Department of Interior's (DOI) Alaska Maritime National Wildlife Refuge.

The Aleutian Archipelago is among the most dynamic ecosystems on Earth, due in part to periodic volcanic eruptions that can eradicate island floras via thick ash deposits (e.g., Talbot et al. 2010). Liverworts are often among the first terrestrial plant species to recolonize perturbed landscapes (Smith \& Griggs 1932), and are known to deliver mycorrhizal inoculum and improve the establishment of vascular plants (Jongmans et al. 2001, Kowal et al. 2016). The biodiversity of liverworts in the Aleutian Archipelago is not well known (Söderström et al. 2015). The primary objectives of this study are to, 1) establish a checklist of the liverwort flora of Attu Island, based on our collections and the collections of other researchers; 2) assign a commonness rating - abundant, common, uncommon, and rare - for each species; 3) record habitat information of each species; and 4) collect voucher specimens of liverwort species. Secondary objectives were to 5) describe the phytogeographic pattern of Attu liverworts with liverwort distribution in circumpolar regions to provide a comparative context, including comparing the liverwort flora of Attu with that of the Commander Islands. The list also provides baseline data for detecting floristic shifts due to impacts of climate change, including generally predicted warming air and ocean temperatures and accompanying changes in precipitation, as well as synergistic interactions among specific factors characteristic of oceanic islands (Harter et al. 2015), including here the impact of ash on floras due to periodic volcanic activity characterizing the Aleutian Archipelago (Anderson \& Bank 1952, Heusser 1990). This research thus informs the DOI regarding biodiversity on lands that the bureau manages. Because the Commander Islands fall within the Commander Islands Biosphere Reserve and is administered by the Ministry of Natural Resources of the Russian Federation, this research provides the Ministry with valuable comparative biodiversity data as well.

A total of 267 liverwort species are reported for Alas$\mathrm{ka}$, excluding the Aleutian Islands, but given the relatively poor historical focus on Alaska liverworts and despite their ecological significance and the richness of the Alaska landscape, it is likely a number of new records are yet to be reported (Söderström et al. (2015). Liverwort collections are reported from Attu Island by Clark \& Frye (1948, 1949), Davison (1993), Evans (1900), Frye \& Clark (1946), Hong (1980), Konstantinova \& Mamontov (2010), Macoun (1902), Persson $(1952,1968)$ and Sharp \& Hattori (1968). In total these reports list approximately 84 species for Attu Island (Davison 1993).

\section{STUDY AREA}

The Aleutian Islands are composed of a chain of sedimentary islands, eroded from older volcanic formations, and capped by steep volcanoes (Gallant et al. 1995). Attu Island is located in the westernmost Aleutian Islands governed by the United States, at approximately $52.83^{\circ} \mathrm{N}$, $173.18^{\circ} \mathrm{E}$ (Fig. 1); Attu Island was extensively glaciated during late Quaternary time, probably during the Wiscon-

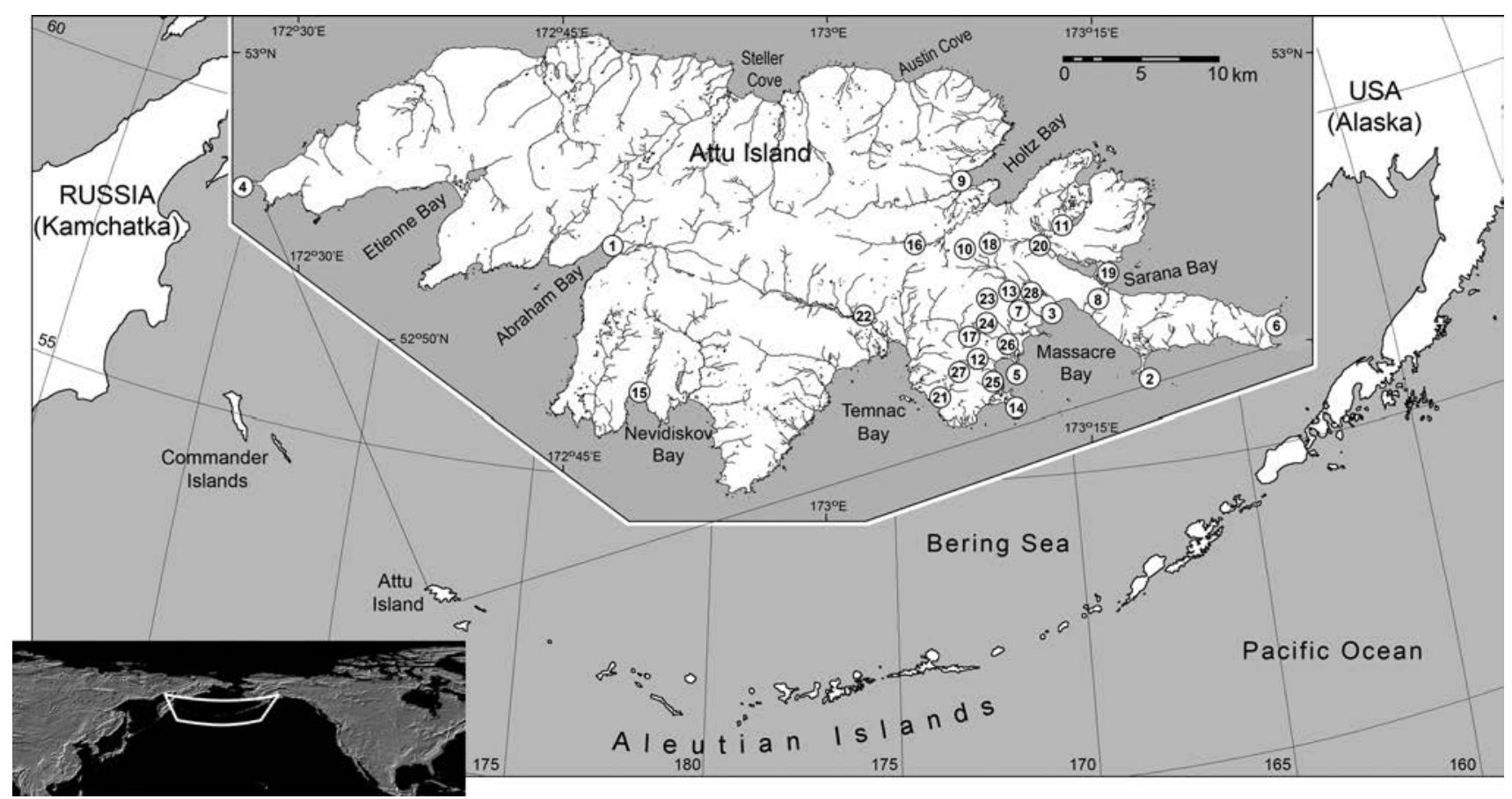

Figure 1 Location of Attu Island, Alaska, and map of the liverwort collection sites. Circled numbers $(1-28)$ are collection sites and listed in the Methods section 
sinan Stage (Gates et al. 1971). The Commander Islands of Russia form a part of the Aleutian Islands with Bering Island $\left(55.02^{\circ} \mathrm{N}, 165.98^{\circ} \mathrm{E}\right)$ and neighboring Copper Island. The geosystems of Bering Island may be divided into three types: plain, mountain and coastal (Ivanov 2003); this classification may be similarly applied to Attu Island. Based on geological evidence (Tsvetlkov et al. 2010), the Commander Islands are older than more eastern islands of the Aleutian Archipelago; evidence of volcanism in the Commander Islands are dated to the Miocene.

A maritime climate prevails throughout the region. The ecoclimatic-phytogeographical system of Tuhkanen (1984) characterizes the Aleutian Islands as northern boreal, hyperoceanic, and perhumid. Mean annual temperature and precipitation of Attu are $3.8^{\circ} \mathrm{C}$ and $1341 \mathrm{~mm}$, respectively (Fig. 2). An alternative climatic classification, Patric \& Black (1968) classify the climate of the Aleutian Islands according to Thornthwaite's system (Thornthwaite 1948) as AC'2rc', that is perhumid, warm microthermal, no season of rainfall deficit, temperature efficiency normal to warm microthermal. A multivariate analysis of climate along the southern coast of Alaska by Farr \& Hard (1987) indicate the Aleutian Islands form a distinct coastal climatic group with moderately cool temperatures in the fall, winter and spring and much colder summers than other coastal stations. In terms of oceanity, Attu is relatively more hyperoceanic $\left(\mathrm{O}_{3}\right)$ than Bering ( $\mathrm{O}_{2}$; Tuhkanen 1984).

General accounts of the vascular flora and vegetation of the Aleutian Islands are provided among others by Bank (1951), Gallant et al (1995), Hultén (1960, 1968), and Tatewaki \& Kobayashi (1934). Information on the vegetation of Attu Island is rather limited; Heusser (1990) published on the late Quaternary vegetation and Talbot \& Talbot (1994) described the coastal vegetation with quantitative comparisons to Aleutian vegetation types described by others. The comparison pointed to a close relationship to the beach and beach-meadow types of the Aleutian Islands and Kamchatka. In a similar manner Krestov (2004) compared the vegetation of the Commander Islands to Attu Island, and other Aleutian Islands and Beringian locations. As stated by Krestov et al. (2010) the archipelagos of the Aleutian, Kuril and Japanese Islands are typified by the presence of plant communities derived from the pre-Pleistocene humid Beringian vegetation complex, which was named Hultenia by Tatewaki (1963).

A factor of major importance in the Aleutians is volcanism as indicated by the thickness, distribution and frequency of tephra layers numbering five on Attu. These recurrent volcanic eruptions with widespread accompanying ash falls influence vegetation composition and distribution (Heusser 1990).

\section{METHODS}

Collections were made by Sandra Looman Talbot (SLT), Stephen S. Talbot (SST) and Wil- fred B. Schofield (WBS) during four periods: 1) July-August 1988 (SLT and SST), 2) August 2000 (SLT, SST, \& WBS), 3) September 2002 (SST \& WBS) and 4) August 2003 (SST). All specimens are vouchered at UBC with partial sets deposited at DUKE and PRC.

Major collection sites are shown in Fig. 1, and are listed below by site number (1-28) followed by a general geographic descriptive name, latitude, longitude, and elevation.

1. Abraham Bay, $52^{\circ} 53^{\prime} \mathrm{N}, 172^{\circ} 47^{\prime} \mathrm{E} ; 5 \mathrm{~m}$;

2. Alexai Point, $52^{\circ} 51^{\prime} \mathrm{N}, 173^{\circ} 14^{\prime} \mathrm{E} ; 195 \mathrm{~m}$;

3. Beach Road, $52^{\circ} 50^{\prime} \mathrm{N}, 173^{\circ} 09^{\prime} \mathrm{E} ; 5 \mathrm{~m}$;

4. Cape Wrangell, $52^{\circ} 56^{\prime} \mathrm{N}, 172^{\circ} 27^{\prime} \mathrm{E}$; $195-488 \mathrm{~m}$;

5. Casco Point, $52^{\circ} 49^{\prime} \mathrm{N}, 173^{\circ} 10^{\prime} \mathrm{E} ; 5 \mathrm{~m}$;

6. Chirikof Point, $52^{\circ} 51^{\prime} \mathrm{N}, 173^{\circ} 24^{\prime} \mathrm{E} ; 6 \mathrm{~m}$;

7. Coast Artillery Hill, $52^{\circ} 51^{\prime} \mathrm{N}, 173^{\circ} 10^{\prime} \mathrm{E} ; 122-152 \mathrm{~m}$;

8. Gilbert Ridge, $52^{\circ} 51^{\prime} \mathrm{N}, 173^{\circ} 14^{\prime} \mathrm{E}$; $30-122 \mathrm{~m}$;

9. Holtz Bay, West Arm, $52^{\circ} 55^{\prime} \mathrm{N}, 173^{\circ} 07^{\prime} \mathrm{E} ; 12 \mathrm{~m}$;

10 Jarmin Pass, $52^{\circ} 58^{\prime} \mathrm{N}, 173^{\circ} 08^{\prime} \mathrm{E} ; 213-610 \mathrm{~m}$;

11. Jim Fish Creek, $52^{\circ} 54^{\prime} \mathrm{N}, 173^{\circ} 14^{\prime} \mathrm{E} ; 61 \mathrm{~m}$;

12. Kingfisher Creek, $52^{\circ} 49^{\prime} \mathrm{N}, 172^{\circ} 09^{\prime} \mathrm{E} ; 30-100 \mathrm{~m}$;

13. Lake Elwood, $52^{\circ} 52^{\prime} \mathrm{N}, 173^{\circ} 10^{\prime} \mathrm{E} ; 12 \mathrm{~m}$;

14. Murder Point, $52^{\circ} 48^{\prime} \mathrm{N}, 173^{\circ} 10^{\prime} \mathrm{E} ; 10-30 \mathrm{~m}$;

15. Nevidiskov Creek (West Arm Nevidiskov Bay), $52^{\circ} 48^{\prime} \mathrm{N}, 172^{\circ} 48^{\prime} \mathrm{E} ; 244-457 \mathrm{~m}$;

16. O’Donnell Valley, $52^{\circ} 54^{\prime} \mathrm{N}, 173^{\circ} 08^{\prime} \mathrm{E} ; 20-30 \mathrm{~m}$;

17. Peaceful Valley, $52^{\circ} 50^{\prime} \mathrm{N}, 173^{\circ} 08^{\prime} \mathrm{E} ; 30-46 \mathrm{~m}$;

18. Robinson Ridge, $52^{\circ} 53^{\prime} \mathrm{N}, 173^{\circ} 09^{\prime} \mathrm{E}$; 396-405 m;

19. Sarana Bay, $52^{\circ} 51^{\prime} \mathrm{N}, 173^{\circ} 23^{\prime} \mathrm{E} ; 11 \mathrm{~m}$;

20. Siddens Valley, $52^{\circ} 52^{\prime} \mathrm{N}, 173^{\circ} 14^{\prime} \mathrm{E}$; ca $10-30 \mathrm{~m}$;

21. Sonam Beach, $52^{\circ} 48^{\prime} \mathrm{N}, 173^{\circ} 06^{\prime} \mathrm{E} ; 15 \mathrm{~m}$;

22. Temnac Valley, $52^{\circ} 50^{\prime} \mathrm{N}, 173^{\circ} 03^{\prime} \mathrm{E}$; 6-472 m;

23. Terrible Mountain, $52^{\circ} 51^{\prime} \mathrm{N}, 173^{\circ} 09^{\prime} \mathrm{E}$; $61-273 \mathrm{~m}$;

24. Thirty-Seven Hill, $52^{\circ} 50^{\prime} \mathrm{N}, 173^{\circ} 09^{\prime} \mathrm{E}$; $50-189 \mathrm{~m}$;

25. USCG Loran A Station, $52^{\circ} 50^{\prime} \mathrm{N}, 173^{\circ} 10^{\prime} \mathrm{E} ; 10-30 \mathrm{~m}$;

26. USCG Loran C Station, $52^{\circ} 50^{\prime} \mathrm{N}, 173^{\circ} 04^{\prime} \mathrm{E} ; 20-50 \mathrm{~m}$;

27. West Mountains, $52^{\circ} 49^{\prime} \mathrm{N}, 173^{\circ} 08^{\prime} \mathrm{E} ; 50-579 \mathrm{~m}$;

28. West Massacre Valley (Henderson River), $52^{\circ} 50^{\prime} \mathrm{N}$, $172^{\circ} 50^{\prime} \mathrm{E} ; 20-60 \mathrm{~m}$.

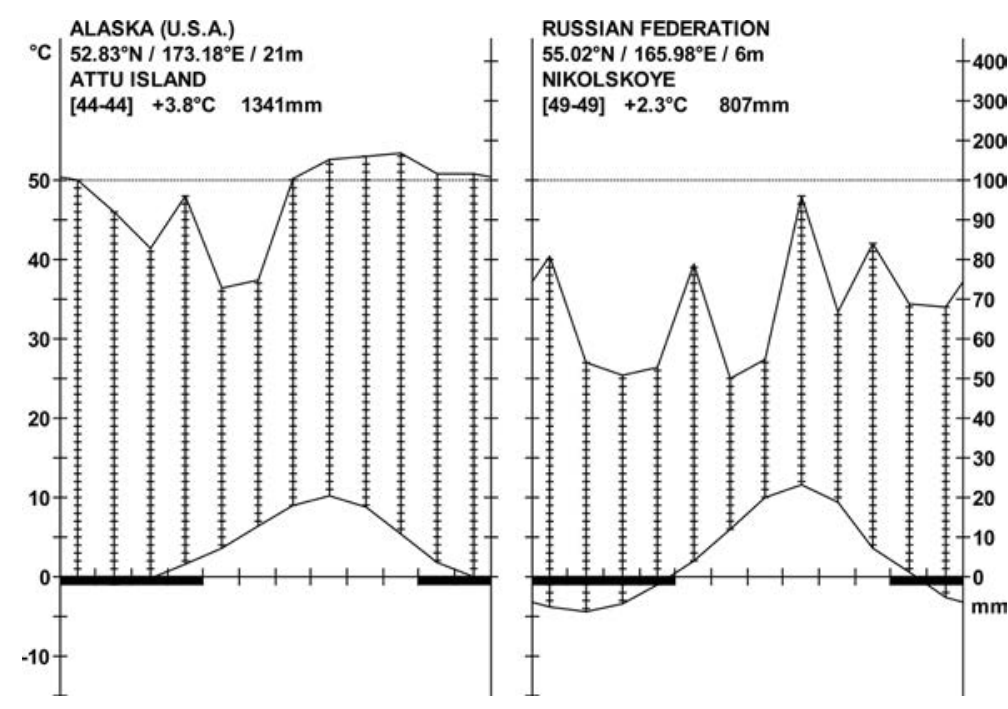

Figure 2 Climate diagrams for Attu Island, Alaska, and Nikolskoye, Bering Island, Russia. Abscissa shows the months January to December. Ordinate: the first dashmark upwards represents $10^{\circ} \mathrm{C}$ and $20 \mathrm{~mm}$, respectively. Name of station given in upper left-hand corner with latitude/longitude and elevation given below. Number of years data were collected for temperature and precipitation given in brackets, followed by mean annual temperature and mean annual precipitation. Top curve is mean monthly precipitation and lower curve is mean monthly temperature. Climate diagram prepared using Lieth et al (1999) based on data Leslie (1989) 
We sought to collect from a spectrum of habitats from seashore to mountain summits (Fig. 3); these habitats included rocky and sandy shores, beach meadows and dunes, mesic forb and graminoid meadows, fern meadows, dwarf shrub heaths, alpine fellfields, mires, ponds and streams, snowbeds, rock cliffs and crevices. For each species we estimated commonness using the classes of Hickman (1993): (1) abundant; very likely to be encountered; nearly always found in appropriate habitats, sometimes forming dense stands; (2) common; likely to be encountered; (3) uncommon; unlikely to be encountered and sometimes not present in appropriate habitats; and (4) rare; extremely unlikely to be encountered, often not present in appropriate habitats, and often restricted to a small number of sites.

The commonness class is followed by habitat information. In turn these data are followed by collection numbers; those numbers starting with the alpha letters "AT" are those of SST and SLT, while all others are those of WBS. Literature citations are given for each species previously reported in the Aleutian Island. Site location names follow. Species reported for the Commander Islands are then given with a literature citation(s).

To assess hepatic distribution within the North Holarctic we assigned the categories of Konstantinova (2000) to each species: I. Arctic: a. Circumpolar, b. Subcircumpolar, c. East Siberian-American-Groenlandic, d. Siberian-American, e. Siberian-West American, f. Chukchi-American-Groenlandic, g. West American, h. Unclear areal; II. Arctomontane: a. Circumpolar, b. Subcircumpolar, c. Amphioceanic, d. Disjunctive, e. Euro-Siberian, f. Eurasian-Groenlandic, g. EurasianGroenlandic-West American, h. Eurasian-West American, k. Siberian-West American, 1. Unclear areal; III. Montane: a. Circumpolar, b. Subcircumpolar, c. Amphioceanic, d. Subatlantic, e. Pacific, f. Disjunctive, g. Euro-West American, h. Euro-Groenlandic, i. American-Groenlandic, k. European, 1. Unclear areal; IV. Arctoboreomontane: a. Circumpolar, b. Subcircumpolar, c. Amphioceanic, d. Disjunctive e. Unclear areal; V. Boreal: a. Circumpolar, b. Subcircumpolar, c. Subatlantic, d. European, e. Euro-American, f. Euro-Groenlandic, g. Disjunctive; VI. Temperate: a. Circumpolar, b. Amphioceanic, c. Pacific, d. Euro-American, e. Eurasian, f. European, g. Unclear areal; VII. Cosmoplitan; and VIII. Unclear distribution. Unassigned species were assigned a category using Bakalin (2010a) and Damsholt (2002).

With the exception of species determined by WBS, for which no indication is given, the determiner's name is indicated by initials, which follows the collection number(s): BS (Blanka Shaw), PD (Paul Davison), Kathrin Feldberg (KF), Olivia Lee (OL) and Jiří Váňa (JV).

Complete information, including detailed collection site descriptions, site locations, latitudes, longitudes, elevations, habitat, collection date, quadrangle map, and determiners, are available at UBC.

\section{RES U LTS}

\section{Annotated catalog of liverworts}

In the following checklist, species reported from Attu are arranged in alphabetical order. Bryophyte nomenclature follows Söderström et al. (2016) for liverworts. An asterisk indi- cates a species newly reported for Attu Island, two asterisks indicate species new to the Aleutian Islands, and three asterisks indicate species new to Alaska. Each species is followed by a series of descriptors: synonyms [in brackets], commonness, habitat, collection number, collection site, previous reports documenting presence on Attu, and geographical distribution, including in some cases distribution elsewhere.

We list 112 liverworts from Attu Island. The number of Attu Island liverwort species equals that of the Commander Islands, where approximately 112 species are reported (Bakalin 2010a). Thirty-four liverwort species (30\% of the Attu flora) are newly reported for Attu and one, Herbertus delavayi, is new to the Aleutian Islands and Alaska. Approximately 66 liverwort species occur in both the Commander Islands and Attu Island; the Attu flora includes 40 species (58 \%) that occur on both Bering and Attu Islands.

\section{Acrobolbus ciliatus (Mitt.) Schiffn.}

Rare; shaded wall, humus hole in gully, 116047A (mixed with Diplophyllum taxifolium)(BS); reported by Sharp \& Hattori (1968); Schofield (2002) noted this species is confined to Alaska to two localities: Baranof Island in southeast Alaska and Attu Island in the Aleutians; Peaceful Valley; IIe.

\section{Anastrepta orcadensis (Hook.) Schiffn.}

Rare; side of humus hole on slope, sedge wetland; 115667 (mixed with Diplophyllum albicans) (BS), 115992 (BS); reported by Persson (1946); Lake Elwood, Terrible Mountain; This species is confined to humid areas near the coast in the Aleutians, southeast Alaska, and neighboring British Columbia (Schofield 2002). IIIf.

\section{Aneura pinguis (L.) Dumort.}

Uncommon; damp cliff on humus; AT225, 115569, 116335, 120246; reported by Clark \& Frye (1948); Casco Point, Murder Point, Thirty-Seven Hill; Bering Island (Bakalin 2005); VII (bipolar).

\section{Anthelia julacea (L.) Dumort.}

Abundant; seepage slope near outcrop, wet late snow patch area; AT 26-27, 115257, 115491A, 115516, 115777, 115816, 115954, 120169, 120394; Davison (1993) reported a specimen collected by Sharp 9023; Beach Road, Casco Point, Coast Artillery Hill, Massacre Bay, Robinson Ridge, Terrible Mountain, Thirty-Seven Hill; Bering Island (Bakalin 2005); IIb.

\section{*Anthelia juratzkana (Limpr.) Trevis.}

Abundant; dryish tundra crusts; fellfield; AT 385, AT 88889, AT18-41, AT26-24, AT27-34, 115516; Cape Wrangell, Jarmin Pass, Robinson Ridge, Thirty-Seven Hill, West Mountains; Bering Island (Bakalin 2005); IIa.

Barbilophozia barbata (Schmidel ex Schreb.) Loeske [Lophozia barbata (Schmidel) Dumort.]

Reported by Persson (1952); IVa (bipolar).

\section{Barbilophozia lycopodioides (Wallr.) Loeske [Lophozia lycopodioides (Wallr.) Cogn.]}

Rare; Nootka lupine meadow, crowberry heath, bank of streamlet, fellfield at mountain summit, Fleabane meadow, open area near road; AT48-49, AT49-36, AT62-41, AT64-38, AT88-756, 116292 (BS), 120122, 120384; reported by Clark \& Frye (1948); Murder Point, Peaceful Valley, Robinson Ridge, Terrible Mountain; Bering Island (Bakalin 2005); IVa.

*Barbilophozia sudetica (Nees ex Huebener) L. Söderstr., De Roo et Hedd. [Lophozia sudetica (Nees ex Huebener) Grolle]

Common; dark strands overgrowing Sphagnum in tundra, Sphagnum at edge of pond base of terrace bank, bank of 

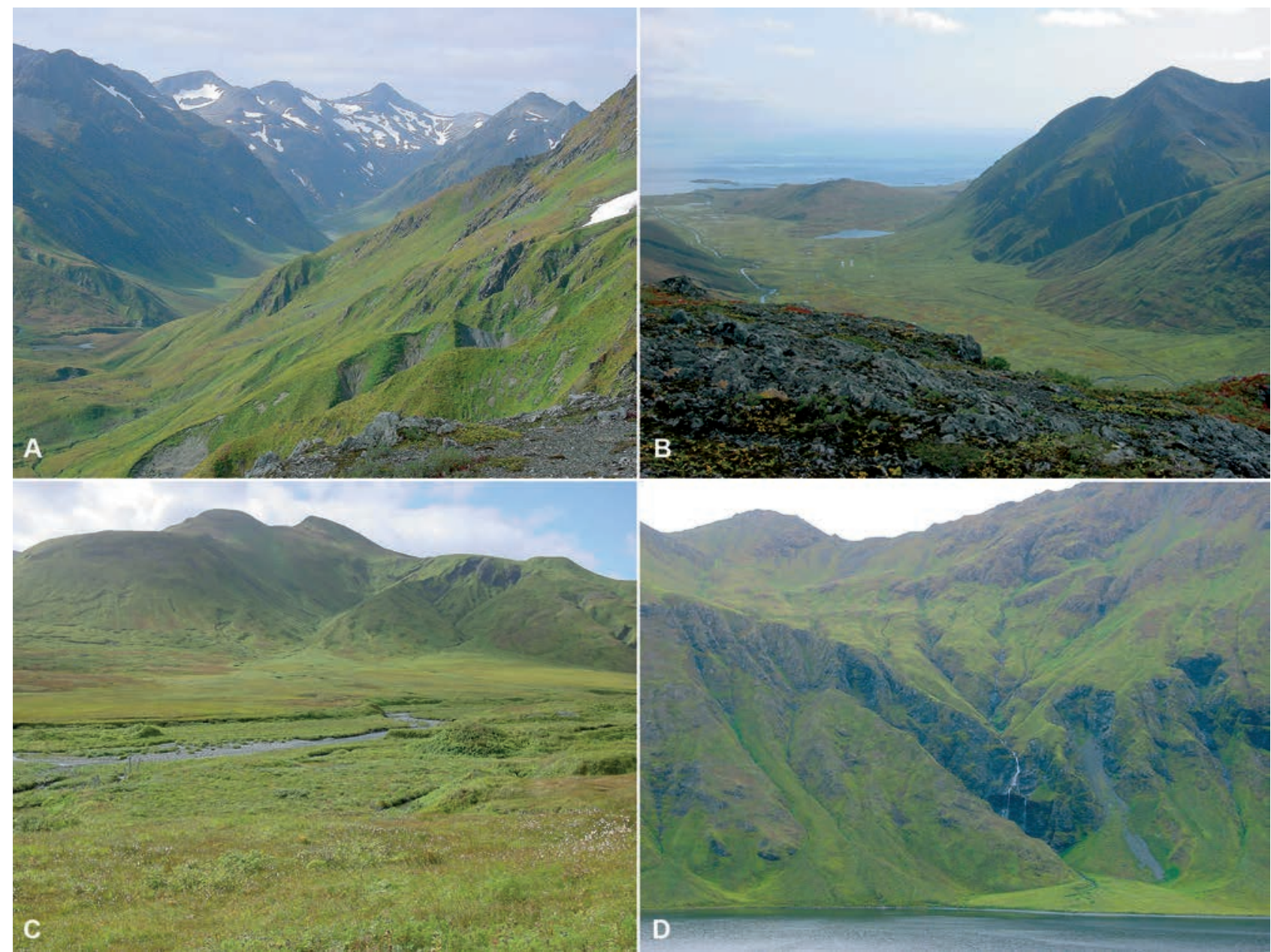

Figure 3 Major habitat types of Attu Island: A - view from eastern Attu Island of the snowbed-rich Attu Mountain area, 18.09.2002. Convex ridge landforms are dominated by dwarf-shrubs and concave depressions are dominated by graminoid and forb meadows. B - view from a fellfield on Gilbert Ridge, Attu Island, looking southeast along West Massacre Valley toward Massacre Bay, 18.09.2002. Terrible Mountain (elevation ca. $610 \mathrm{~m}$ ) is on the right with Coast Artillery Hill (elevation $168 \mathrm{~m}$ ) and Lake Elwood in the distance. The valley plain is dominated by mires of Lyngbye sedges and tall cottongrass. C - view southwest from Peaceful River toward Kingfisher Creek in the Weston Mountains (elevation ca $488 \mathrm{~m}$ ), Attu Island, Alaska, 13.09.2002. Forb meadows dominate riparian sites and sedge mires occupy the toe slopes of the mountains. Mountain slopes are covered with a mosaic of meadows and dwarf shrub communities. D - view of the north slope of Gilbert Ridge (elevation ca $460 \mathrm{~m}$ ) above Lake Nicolas in Siddens Valley, Attu Island, Alaska, 21.09.2002. Soils are typically saturated

streamlet, slope of late snow area, outcrop face in Poblia, old pier, damp humus on cliff shelf, alpine fellfield; AT1719 (JV), 115374 (BS), 115398 (BS), 115642 (BS), 115704 (mixed with Diplophyllum albicans, Bazzania tricrenata) (BS), 115739 (BS), 115838 (BS), 115842 (BS), 115984 (BS), 116159 (BS), 116160 (BS), 116299 (BS); Alexai Point, Coast Artillery Hill, Gilbert Ridge, Jarmin Pass, Murder Point, Robinson Ridge, Terrible Mountain, USCG Loran C Station; Bering Island (Bakalin 2005); IIa.

\section{Bazzania tricrenata (Wahlenb.) Lindb.}

Rare; shaded bank, damp bank in gully, subalpine fleabane meadow, shaded humus hole, gully walls, mosses on streamlet bank, damp slope near cliff base; mixed with Diplophyllum albicans, Marsupella emarginata, Herbertus cf. dicranus; AT241, 115382, 115613, 115676, 115685A, 115690, 115840, 115855 (BS), 116005, 116043, 116197, 116208 (BS), 116232; reported by Clark \& Frye (1949); Coast Artillery Hill, Gilbert Ridge, Lake Elwood, Peaceful Valley, Terrible Mountain, Thirty-Seven Hill, West Mountains; IIIb.

\section{*Bazzania trilobata (L.) Gray}

Uncommon; bank on mire slope, Sphagnum compactum mesic mire; AT25-35, 115601; Alexai Point, Peaceful Valley; VIb.

\section{Blasia pusilla L.}

Rare; lava cliff; shaded humid base of cliff; 124809; reported by Clark \& Frye (1948); USCG Loran A Station; Bering Island (Bakalin 2005); VII (bipolar).

\section{Blepharostoma trichophyllum (L.) Dumort.}

Common; rock outcrop, shaded cliff, cliff crevices, Nootka lupine meadow, shaded bank, shaded side of hole on slope; AT19-33, AT389, 115328, 115465, 115548, 115609, 115650, 115848, 115943A, 116273, 120222, 120429; reported by Clark \& Frye (1949) and Sharp \& Hattori (1968); Beach Road, Coast Artillery Hill, Cape Wrangell, Casco Point, Jarmin Pass, Peaceful Valley, Robinson Ridge, Thirty-Seven Hill; Bering Island (Bakalin 2005); IVa (bipolar).

\section{* Calycularia laxa Lindb. \& Arnell}

Uncommon; sedge wet meadow, rock outcrop, shaded cliff shelf, under boulder; AT1-27, AT88-698 (PDPD), AT88707 (PD), AT88-1168 (PD), AT88-709, 115519, 124125A; this species was originally determined as Calycularia crispula Mitten (Davison \& Smith 1992), but see Konstantinova \& Mamontov (2010) who indicate it is C. laxa and should be updated; Jarmin Pass, Nevidiskov Creek, O’Donnell Valley, 
Thirty-Seven Hill; Copper Island (Bakalin \& Cherdantseva 2008); Id (Note: distribution category corrected from Ie as it occurs in both Siberia and America).

Calypogeia azurea Stotler \& Crotz [C. trichomanis auct.; not (L.) Corda]

Reported as C. trichomanis by Persson (1952); the identification may be based on dry material and might be doubtful; VIb.

\section{Calypogeia muelleriana (Schiff.) Müll. Frib.}

Common; snowbed meadow, wet sedge meadow, ladyfern meadow, shaded humus hole, Sphagnum compactum tuft; AT4-44, AT41-21, AT39-38, AT42-33, AT43-26, AT4345, AT49-49, AT61-16, 115371 (mixed with Pellia neesiana) (BS), 115673, 115798 (mixed with Diplophyllum albicans)(BS) 115822 (mixed with Lophozia sp.) (BS), 115910 (mixed with Scapania uliginosa)(BS); reported by Persson (1968); Coast Artillery Hill, Jarmin Pass, O’Donnell Valley, Peaceful Valley, Robinson Ridge, Terrible Mountain, West Massacre Valley; Bering and Copper islands (Bakalin 2005); IVa.

\section{*Calypogeia sphagnicola (Arnell \& J. Perss.) Warnst. \& Loeske}

Rare; wet sedge meadow, hard hummocks on damp slope, Sphagnum hummock on bank; AT42-38, 115640 (mixed with Rhizomnium punctatum, Cephalozia bicuspidata), 115280A (BS), 116051 (BS), 120303; West Massacre Valley, Jarmin Pass, Peaceful Valley, West Mountains; Bering Island (Bakalin 2005); IVa (bipolar).

* Cephalozia ambigua C. Massal. [Cephalozia bicuspidata subsp. ambigua (C. Massal.) R.M. Schust.]

Rare; alpine blueberry-crowberry meadow; AT33-43 (BS), 115244; Coast Artillery Hill; Bering Island (Bakalin 2005); IIa.

Species separated from $C$. bicuspidata on the basis of smaller leaf cells and different chromosome number. Recent studies based on molecular data (Vilnet et al. 2012, Feldberg et al. 2016) showed that this species nests in the robust $C$. bicuspidata clade and probably it is should be not separated on species level. But the whole C. bicuspidata complex (C. bicuspidata clade) is very complicated and needs further detailed study to clarify if the specific status of some taxa of this complex is correct.

\section{Cephalozia bicuspidata (L.) Dumort.}

Common; shaded humus bank and hole, damp outcrop, wet shaded base of cliff, Sphagnum compactum tuft, sedge wetland, wet late snow area, damp humus bank in gully, mesic mire, Nootka lupine meadow; 115366 (mixed with Diplophyllum albicans, Kurvia pauciflora) (BS), AT25-37 (JV), AT39-40 (mixed with Calypogeia sphagnicola)(BS), AT42-39 (mixed with Calypogeia muelleriana, Scapania cf. irrigua, Harpanthus flotovianus), AT42-40 (BS), AT60-21 (BS), AT60-28 (BS), AT61-28, AT62-61 (mixed with Trilophoria quinquedentata)(BS), 115383 (BS), 115555, mixed with Diplophyllum albicans, Gymnocolea inflata, Nardia scalaris, Barbilophozia sudetica (JV), 115799 (mixed with Diplophyllum albicans)(BS), 115879 (BS), 115993 (mixed with Gymnocolea inflata)(BS), 116161 (JV), 116170 (mixed with Fuscocephaloziopsis albescens)(JV), 116262 (BS); reported by Clark \& Frye (1948), Evans (1900), Macoun (1902); Alexai Point, Casco Point, Coast Artillery Hill, Lake Elwood, O'Donnell Valley, Robinson Ridge, Terrible Mountain, West Massacre Valley; Bering Island (Bakalin 2005); VII (bipolar).

Cephaloziella divaricata (Sm.) Schiffn. [Cephaloziella byssacea Warnst.]

Rare; earth of damp area, damp cliff shelf; 115394 (BS), 115562 (JV), 115802 (BS); reported by Clark \& Frye (1948); Coast Artillery Hill, Terrible Mountain; Bering Island (Bakalin 2005); IVa (bipolar).

*Cephaloziella varians (Gottsche) Steph.

Rare; damp area near stream, shaded damp humus of cre- vice; 116057A (JV), 116310 (BS); Murder Point, Peaceful Valley; IIa (bipolar).

\section{Chiloscyphus pallescens (Ehrh.) Dumort.}

Rare; floating in small pool of gully, narcissus anemone meadow, subalpine fleabane meadow, humus in gully; AT12-31, AT64-43, 116191 (BS), 116319 (JV); reported by Clark \& Frye (1948, 1949), Persson (1952); Thirty-Seven Hill; Bering Island (Bakalin 2005); Va.

\section{Chiloscyphus polyanthos (L.) Corda}

Uncommon; Nootka lupine alluvial meadow, mountain streamlet, edge of pond in wetland, bank above streamlet, alluvial meadow; 50-44 (mixed with Conocephalum salebrosum), AT51-42, AT345, AT346, AT2000-50-44 (BS),115623, 115991, 116068, 116321; reported by Clark \& Frye (1948); Cape Wrangell, Lake Elwood, Murder Point, Peaceful Valley, Siddens Valley, Terrible Mountain; Bering Island (Bakalin 2005); Va.

\section{Conocephalum salebrosum Szweyk., Buczk. et Odrzyk.}

Abundant; ladyfern meadow, eared Indian plantain meadow, damp area near stream, shaded area by outcrop; AT13-18, AT19-20, AT65-18, AT71-21, AT102-39, AT344, 115620, 115297, 116300; reported by Clark \& Frye (1948, 1949), Frye \& Clark (1946); Cape Wrangell, Murder Point, Sarana Bay, Sonam Beach, West Arm Holtz Bay, West Mountains; Bering Island (Bakalin 2005); Va.

\section{Diplophyllum albicans (L.) Dumort.}

Uncommon; margin of pool in crowberry mire, humus bank, shaded earth bank, damp cliff, deep shaded humus hole, base of outcrop on ridge, soil in rock crevice; AT881066, AT88-1067, AT88-1072, AT136, AT140, AT419, AT234 (BS), 115324A, 115364, 115389, 115606, 115707, 115889, 116129 (mixed with Sphenolobus minutus)(BS), 116352, 120422, 120310A; reported by Clark \& Frye (1948, 1949), Frye \& Clark (1946); Cape Wrangell, Casco Cove, Henderson River, Jarmin Pass, Massacre Bay, Peaceful Valley, Robinson Ridge, Temnac Cove, Terrible Mountain; Bering Island (Bakalin 2005); IIIc.

\section{Diplophyllum obtusifolium (Hook.) Dumort.}

Reported by Clark \& Frye (1948), Persson (1952); IVb.

\section{Diplophyllum taxifolium (Wahlenb.) Dumort.}

Rare; rock outcrop, shaded cliff crevices, cliff ledge, walls of humus hole in gully; AT716, 115499A (mixed with Diplophyllum albicans, Radula sp.)(BS), 115512B, 115544B, 115552A, 116047, 116231 (mixed with Trilophozia quinquedentata, Marsupella emarginata), 120506A; reported by Clark \& Frye (1948, 1949), Persson (1952), Sharp \& Hattori (1968); Casco Point, Nevidiskov Bay, Peaceful Valley, Thirty-Seven Hill; Bering Island (Bakalin 2005); IVa.

\section{Douinia imbricata (M. Howe) Konstant. \& Vilnet [Diplophyllum imbricatum (M.A. Howe) Müller Frib.]}

Rare; open humus of cliff shelf, Sphagnum compactum mesic mire; AT25-33, 115952 (OL); reported by Hong (1980); Alexai Point, Beach Road; Bering Island (Bakalin 2005); IIIe.

\section{Douinia plicata (Lindb.) Konstant. \& Vilnet [Diplophyl- lum plicatum Lindb.]}

Rare; humus on cliff shelf, mesic mire, bog blueberry heath, steep hillside tundra, bank above streamlet gully, crowberry heath, tundra bank, side of herb-shaded gully, many-flowered sedge mire; AT21-27, AT22-30, AT24-27, AT103-29, 115586, 115691, 115952 (mixed with Diplophyllum taxifolium), 120170, 120310B, 120343; reported by Sharp \& Hattori (1968); Beach Road, Casco Cove, Coast Artillery Hill, Gilbert Ridge, Terrible Mountain, West Mountains; Bering Island (Bakalin 2005); IIIe. 
*Frullania nisquallensis Sull. [F. tamarisci subsp. nisquallensis (Sull.) S. Hatt., F. tamarisci var. nisquallensis (Sull.) S. Hatt.]

Rare; growing up cliff base, shaded cliff, verified genetically; 115271(GenBank Accession No. MH325471), 115424 (GenBank Accession No. MH325472), 116193 (GenBank Accession No. MH32570); Massacre Bay, Thirty-Seven Hill; IIk.

\section{Fuscocephaloziopsis albescens (Hook.) Váňa \& L.}

Söderstr. [Pleurocladula albescens (Hook.) Spruce]

Rare; rock cliff outcrop, late snowbed meadow, cliff crevice, mesic mire, seepage streamlet; AT20-33, AT22-40, AT59-27 (mixed with Anthelia juratrkana), AT60-13, AT414, AT477A, 116147, 116179 (mixed with Moerckia blyttii)(JV), 116265, 120172; reported by Davison (1993) from a collection of Sharp 9546 (TENN); Cape Wrangell, Coast Artillery Hill, Robinson Ridge, Terrible Mountain; Bering Island (Bakalin 2005); IIa.

\section{Fuscocephaloziopsis lunulifolia (Dumort.) Váňa \& L. Söderstr. [Cephalozia lunulifolia (Dumort.) Dumort.]}

Rare; shaded bank of gully, humus in maritime outcrop; mixed with Kurzia pauciflora, Cephalozia bicuspidata, Diplophyllum taxifolium; 115677 (BS), 116313 (BS); reported by Clark \& Frye (1949), Sharp \& Hattori (1968); Alexai Point, Murder Point; Bering Island (Bakalin 2005) and Copper Island (Bakalin 2010a); IVa.

\section{Fuscocephaloziopsis pleniceps (Austin) Ván̆a \& L.} Söderstr. [Cephalozia pleniceps (Austin) Lindb.]

Uncommon; shaded wall of gully, shaded humus of maritime outcrop, damp bank of river; 115880 (mixed with Cephalozia bicuspidata, Nardia scalaris)(BS), 116220 mixed with Isopterygium cf. pulchellum (JV), 116297 (JV), 116354 (BS \& JV); reported by Persson (1952); Alexai Point, Henderson River, Murder Point, Thirty-Seven Hill; Bering Island (Bakalin 2005); IVa (bipolar).

\section{Gymnocolea inflata (Huds.) Dumort.}

Abundant; bog blueberry mire, submerged in rocky stream, wet sedge seepage at streamlet head, seepage slope near outcrop, undercut pool bank in mire, black mats in streamlet, stream depression; AT42-43, AT44-31, AT88-641, AT88-646, AT88-648, AT88-658, AT88-660, AT88-1074, 115250, 115375, 115716, 115818, 115894A, 120143; reported in Davison (1993) as a collection by Sharp 9067; Abraham Bay, Coast Artillery Hill, Jim Fish Valley, O’Donnell Valley, Peaceful River Valley, Robinson Ridge, Temnac Cove, Thirty-Seven Hill, West Massacre Valley; Bering Island (Bakalin 2005); IVa.

Gymnomitrion alpinum (Gottsche ex Husn.) Schifn. [Marsupella alpina (Gottsche ex Husn.) Bernet]

Reported by Persson (1968); IId.

Gymnomitrion brevissimum (Dumort.) Warnst. [Marsupella brevissima (Dumort.) Grolle]

Rare; blowout in tundra; 116163 (JV); reported by Clark \& Frye (1948) as Gymnomitrium varians; Robinson Ridge; Bering Island (Bakalin 2005); IId.

Gymnomitrion commutatum (Limpr.) Schiffn. [Marsupella commutata (Limpr.) Bernet]

Reported by Davison (1993) from a collection of Sharp 9467 (TENN); IId.

\section{Gymnomitrion concinnatum (Lightf.) Corda}

Common; boulder on slope, cliff crevice, cliff shelf, bog blueberry meadow, outcrop near summit, margin on late melt snowbed, shaded face of outcrop; AT00-33-41, AT130, 115246, 115474, 115490, 115638, 115713, 115714, 115801,115619, 115525, 115664 (BS), 115847 (BS), 116156 (mixed with Diplophyllum albicans)(BS), 116205, 116349, 120158, 120482; reported by Davison (1993) from a collec- tion of Sharp 9404 (TENN); Coast Artillery Hill, Henderson River, Peaceful Valley, Robinson Ridge, Siddens Valley, Temnac Valley, Terrible Mountain, Thirty-Seven Hill; Bering Island (Bakalin 2005); IIa (bipolar).

\section{Gymnomitrion corallioides Nees}

Rare; exposed outcrop on ridge; 115813 (mixed with Gymnomitrion pacificum); reported by Davison (1993) from the collection S. \& S. Talbot 473 (UBC); Coast Artillery Hill; Bering Island (Bakalin 2005); IIa.

\section{Gymnomitrion obtusum Lindb.}

Uncommon; damp open cliff face, dry cliff, damp disturbed area; 115835 (BS), 115906 (BS), 116343 (mixed with Diplophyllum albicans)(BS); reported by Clark \& Frye (1949); Coast Artillery Hill, Jarmin Pass, mouth of Peaceful River; IIIf (bipolar).

\section{* Gymnomitrion pacificum Grolle}

Rare; outcrop on alpine ridge, cliff crevices, exposed alpine gravel, rocky alpine ridge, open tundra; 115708, 115742, 115745, 115849, 115883 (BS \& JV), 116146, 120178, 120392; Alexai Point, Coast Artillery Hill, Robinson Ridge, Terrible Mountain, Thirty-Seven Hill; Bering Island (Bakalin 2005); IIIe.

\section{Gyrothyra underwoodiana M. Howe}

Rare; shaded ditch wall in tundra; 116235; reported by Davison (1993) from the collection Sharp 9551 (TENN); ThirtySeven Hill; VIb (Western North America Pacific Endemic).

\section{*Harpanthus flotovianus (Nees) Nees}

Uncommon; wet cliff, lakeside bank, shaded wall of hole in gully, stream bank, narcissus anemone meadow, shaded wall of hole in gully, alpine spring bank, tall ladyfern meadow, Nootka lupine meadow; AT4-42, AT62-42, 116003 (mixed with Scapania undulata)(BS), 115750,116093 (BS), 116236, 120292, 120348; Beach Road, Casco Bay, Lake Elwood, Peaceful Valley, Terrible Mountain, Thirty-Seven Hill; Bering Island (Bakalin 2005); IVc.

\section{Herbertus aduncus (Dicks.) Gray}

Reported by Davison (1993) from a collection of Sharp 9177 (TENN); This species is more common in the area (Söderström et al. 2015), but this species was incorrectly separated from others in the past; apparently $H$. aduncus is not reported from the Commander Islands (Bakalin \& Cherdandtseva (2008), nor Bakalin (2010a). IIIe.

\section{***Herbertus delavayi Steph.}

Uncommon; floor of deep humus hole in gully, shaded all of gully; 115674 (JV), 115839 (KF), 115840 (JV); Coast Artillery Hill, Terrible Mountain; there is difficulty in placing this taxon in a geographic category as it was once considered identical with $H$. sendtneri and molecular methods may separate it; it is known from Scotland, China, Yunnan, Bhutan, British Columbia (UBC, Schofield 83644) as reported by Heinrichs et al. (2010), and now the Aleutian Islands; VIII. Note: this species is difficult to recognize morphologically; also Juslén (2006) in her monograph of Asiatic Herbertus placed this species based on the type under $H$. sendtneri; based on morphological characteristics this species is in an intermediate position between $H$. sendtneri and $H$. dicranus, but not in molecular characteristics, where it is clearly different. Until now, based on specimens examined by J. Vána and determined by molecular characteristics it is found in Schofield 115839, 115840 (in mixture with Bazzania tricrenata).

*Herbertus dicranus (Gottsche, Lindb. \& Nees) Trevis. Abundant; damp bank of small gully, reddish-rusty mats on damp outcrop of mountain slope, deeply shaded gully, damp outcrop face, seepage slope, intermittent streamlet in tundra, shaded wall of hole on slope, shaded wall of gully, 
steep bank of slope, shaded cliff, humus brow of sod bank, damp slope, dryish bank at upper edge of mesic mire, damp bank of mesic mire, cliff shelves, snowbed; AT1988-135, 115326, 115387, 115581, 115671, 115775, 115868, 115891, 115962, 116004, 116044, 116050, 116131, 120167, 120239, 120345, 120354, 120415; Alexai Point, Beach Road, Coast Artillery Hill, Henderson River, Jarmin Pass, Lake Elwood, Peaceful Valley, Temnac Cove, Terrible Mountain, ThirtySeven Hill, West Mountains; unreported from the Commander Islands (Bakalin 2010a); IIh. [AmphiPacific (disjunct Africa and India)].

\section{*Herbertus sendtneri (Nees) Lindb.}

Uncommon; damp bank of tundra, wall of humus hole of periodic streamlet, subalpine bank of gully, shaded wall of gully, snow bed; AT88-135, 116339 (mixed with Mylia tay lori)(BS); 115378, 115685, 115841, 116339, 120496; Alexai Point, Coast Artillery Hill, Temnac Cove, Terrible Mountain, Thirty-Seven Hill; IIIf.

Note: until now not known from the whole of Africa and in $\mathrm{Eu}-$ rope known only from Austrian Alps. Known from Aleutian Islands, Alaska and British Columbia, the mountains of Central and South America (from Mexico to Bolivia and Brazil), Gough Island, probably also Azores, in Asia from Himalayas (India and Nepal).

\section{Hygrobiella laxifolia (Hook.) Spruce}

Rare; bank of streamlet, wet river bank, wet cliff; 115927, 116056 (BS \& JV), 116342 (mixed with Scapania subalpina) (BS); reported by Frye \& Clark (1946); Jarmin Pass to O’Donnell Valley, Peaceful Valley; Bering Island (Bakalin 2005); IIIc.

\section{Jungermannia atrovirens Dumort.}

Rare; shaded humid cliff; 115760 (JV); reported by Clark \& Frye (1948); Massacre Bay; Bering Island (Bakalin 2005); IIIb.

\section{Jungermannia borealis Damsh. \& Váňa}

Rare; seepage area, blackish masses in wet outcrop; 115344 (JV), 116260 (BS), 116337 (JV); reported by Davison (1993) from a collection of Sharp 9460 (TENN); Murder Point, Terrible Mountain; Bering Island (Bakalin 2005); IIg.

\section{Jungermannia exsertifolia Steph. subsp. cordifolia (Dumort.) Váňa}

Common; stones at bottom of herb shaded gully, shaded bottom of humus hole, seepage slope, black and green floating mat in seep, wet rock in streamlet; 120220, 115264 (JV), 115592 (JV), 115693, 115596 (JV), 116271 (JV); Coast Artillery Hill, Gilbert Ridge, Terrible Mountain, Thirty-Seven Hill, USCG Loran C Station; Bering Island (Bakalin 2005 as Jungermannia eucordifolia Schljakov); IIIa.

\section{*Jungermannia pumila With.}

Rare; wet bank of streamlet; 115683 (JV); Alexai Point; Bering Island (Bakalin 2005); IIa (bipolar).

\section{* Kurzia makinoana (Steph.) Grolle}

Rare; shaded humus hole in tundra; 115386 (mixed with Nardia scalaris)(BS); Terrible Mountain; Bering Island (Bakalin 2005); VIb.

\section{Kurzia pauciflora (Dicks.) Grolle}

Rare; bank above stream, hard bank on mesic slope, peaty sod, shaded hole in slope; 115606A (BS), 115274, 115768 , 115998; reported by Davison (1993) from a collection of Sharp 9475 (TENN); Peaceful Valley, Terrible Mountain, West Mountains (Kingfisher Creek); Vb.

\section{Lepidozia reptans (L.) Dumort.}

Reported by Davison (1993) from a collection of Sharp 9210 (TENN); Va.

\section{*Lophozia murmanica Kaal.}

Rare; hard bank of mesic slope; 115999 (mixed with Shistochilopsis incisa, (JV); Lake Elwood; IVa.

*Lophozia guttulata (Lindb. et Arnell) A. Evans [Lophozia longiflora (Nees) Schiffn. p.p.]

Common; bank of streamlet, wet bank of tarn, shaded cliff shelf; 115629 (mixed with Calypogeia muelleriana, Neoorthocaulis floerker)(BS), 115668 (mixed with Buckiella undulata, Barzania tricrenata, Neoorthocaulis floerkei)(BS), 115808 (BS \& JV); mixed with Diplophyllum albicans), 115853 (BS \& JV), 116113 (JV), 116123, 116272 (JV); Coast Artillery Hill, Henderson Creek, Robinson Ridge, Terrible Mountain; IVa.

\section{Lophozia ventricosa (Dicks.) Dumort.}

Uncommon; cliff shelf, humus in maritime outcrop; 115949 (BS), 116312 (BS); reported by Davison (1993) in Clark \& Frye (1949); Beach Road, Massacre Bay, Murder Point; Bering Island (Bakalin 2005), Copper Island (Bakalin 2009 as Lophozia longiflora); Va.

Lophozia wenzelii (Nees) Steph.

Rare; old timber from derelict building; 116103 (mixed with Ptilidium pulcherrimum)(BS); reported by Davison (1993) from a collection of Sharp 9368 (TENN); Peaceful Valley; Bering Island (Bakalin 2005); IVa.

Lophoziopsis excisa (Dicks.) Konstant \& Villnet [Lophozia excisa (Dicks.) Dumort.]

Reported by Clark \& Frye (1948), Persson (1952); Bering Island (Bakalin 2005); IVa (bipolar).

\section{Marchantia polymorpha L.}

Rare; Lynbye sedge poor fen; AT15-31; reported by Clark \& Frye (1948), Frye \& Clark (1946); Peaceful Valley; Bering Island (Bakalin 2005); VII (bipolar).

*Marsupella apiculata Schiffn. [Gymnomitrion apiculatum (Schiffn.) Müll.Frib.]

Rare; exposed alpine ridge; 115740 (mixed with Gymnomitrion concinnatum (JV); Robinson Ridge; Bering Island (Bakalin 2005); IIa.

* Marsupella aquatica (Lindenb.) Schiffn. [M. emarginata var. aquatica (Lindenb.) Dumort.]

Rare; cliff crevices; 115846 (BS); Coast Artillery Hill; IIIa.

\section{Marsupella emarginata (Ehrh.) Dumort.}

Abundant; seepage in slope, seep in rock crevice, damp cliff shelf, damp cliff, undercut of pool bank in mire, old road bed, shaded cliff crevices, streamlet in slope, wet outcrop, black masses in late snow patch, wet alpine tundra slope, ditch near road, rock cliff outcrop; AT88-603, AT88604, AT88-650, AT88-718a, 413b, 115824A (BS), 115827 (OL), 115828 (BS), 115856 (BS), 115861 (BS) 115924 (BS), 115942 (mixed with Scapania curta)(BS), 115943 (BS), 116096 (BS), 116114 (BS), 116152 (BS), 116166 (JV), 116174 (BS), 116182 (BS); reported by Clark \& Frye (1949); Alexai Point, Beach Road, Cape Wrangell, Chirikof Point, Coast Artillery Hill, Henderson Creek, Jarmin Pass, Jim Fish Valley, Peaceful Valley, Robinson Ridge, West Arm Nevidiskov Bay; Bering Island (Bakalin 2005); IIIa (bipolar).

\section{Marsupella sparsifolia (Lindb.) Dumort.}

Rare; wet seepage depression; 120164; Coast Artillery Hill; Copper Island (Bakalin \& Cherdantseva 2008); IId (bipolar).

Marsupella sphacelata (Giesecke ex Lindenb.) Dumort. Uncommon; late snow melt slope, submerged in pool, cliffs by streamlet, Sphagnum mire, wet slope, old runway pavement, snow-melt slope; AT61-32 (BS), AT88-808 (PD), 115256, 115726 (BS \& JV), 115805 (mixed with Barbilophozia sudetica, Scapania paludosa)(BS), 115887A (BS); re- 
ported by Davison (1993) from a collection of Sharp 9531 (TENN); Alexai Point, Coast Artillery Hill, Jarmin Pass, Peaceful River, Robinson Ridge, O’Donnell Valley, ThirtySeven Hill; IIIb.

Marsupella stableri Spruce [Marsupella boeckii var. stableri R.M. Schuster]

Rare; damp alpine outcrop, streamlet margin, late snowmelt slope, 116165 (OL), 116175 (BS), 120398 (OL), 120464 (OL); reported by Davison (1993) from a collection of Sharp 9505 (TENN); Robinson Ridge, Siddens Valley; Bering Island (Bakalin 2005); IIIf.

\section{Metacalypogeia cordifolia (Steph.) Inoue [Calypogeia cordifolia Steph.]}

Rare; damp shaded cliff near stream; 116306 (mixed with Blepharostoma trichophyllum, Fuscocephaloziopsis cf. lunulifolia) (BS); reported by Davison (1993) from a collection of Sharp 9052 (TENN); Murder Point; VIb.

\section{Metzgeria conjugata Lindb.}

Uncommon; rock outcrop, shaded cliff, damp bank in gully, shaded humid cliff shelves; AT88-708, 115425, 115870, 115896, 116207, 120456; reported by Davison (1993) from the collection S. \& S. Talbot 689 (UBC); Alexai Point, Gilbert Ridge, Jarmin Pass, Thirty-Seven Hill, West Arm Nevisdiskov Bay; Va. Söderström et al. (2010) stated that Metageria conjugata is a complex taxon; accordingly, we are conservative in naming the Attu specimens.

\section{Metzgeria pubescens (Schrank) Raddi [Apometzgeria} pubescens (Schrank) Kuwahara]

Rare; deeply shaded wall of gully; 120355; reported by Frye \& Clark (1946); West Mountains; IIIb (bipolar).

\section{Moerckia blyttii (Mørch) Brockm.}

Uncommon; peaty bank of alpine streamlet, Sphagnum in bog blueberry mire, Pyrenean sedge snowbed meadow, mountain marestail snowbed meadow, peaty bank of pond, bank of streamlet in alpine saddle; AT59-23, AT88-655, 115654, 116179 mixed with Fuscocephaloziopsis albescens (JV), 120155, 120262; reported by Davison (1993) from collection of Sharp 9214; Coast Artillery Hill, Jim Fish Valley, Robinson Ridge, Thirty-Seven Hill; Bering Island (Bakalin 2005); IId.

\section{Moerckia hibernica (Hook.) Gottsche}

Rare; rock cliff outcrop, AT457; reported by Davison (1993) from the collection S. \& S. Talbot 457 (UBC); Cape Wrangell; Vb.

\section{Mylia taylorii (Hook.) Gray}

Common; mire bank, damp bank in tundra, seepy bank of tundra, Sphagnum on tundra slope, caltha-leaf avens meadow, mire bank, seepage slope near outcrop, damp slope near ridge top, ditch by runway, shaded humus hole on slope, Sphagnum compactum mesic mire, Pyrenean sedge snowbed meadow, wall of gully, side of herb-shaded gully, damp bank in tundra, ditch by airport runway; AT2-49, AT2540, AT60-36, 115314, 115380, 115605 (mixed with Sphenolobus saxicola, Herbertus cf. dicranus, Diplophyllum albicans)(BS), 115611, 115657, 115817, 115826, 115388, 115602, 116339 (mixed with Herbertus sendtneri)(BS), 120299, 120307; reported by Davison (1993) from collection of Llano 1383e (UBC); Alexai Point, Coast Artillery Hill, Murder Point, Peaceful Valley, Terrible Mountain, West Mountains; IIIb.

* Nardia breidleri (Limp.) Lindb.

Rare; Alaska arnica - Nootka lupine fellfield; AT26-29; Robinson Ridge; Bering Island (Bakalin 2005); IId.

*Nardia geoscyphus (De Not.) Lindb.

Rare; shaded side of hole in slope, peat moss tuft in mesic mire; 115649 (BS \& JV), 115658; Robinson Ridge; Bering Island (Bakalin 2005); IIa.

*Nardia japonica Steph.

Rare; damp outcrop; 115919 (BS); Jarmin Pass; Bering Island (Bakalin 2005); IIh.

\section{Nardia scalaris Gray}

Abundant; damp slope at head of streamlet, open damp area in ridge, earth bank, earth base of huge boulder, wet cliff, rock outcrop, undercut pool bank in alpine blueberry mire, mineral soil in rock crevice, damp edge of stream, shaded earth bank, humus clods on barren ridge, wet bank above stream, shaded cliff shelves, sand dune, snowbed, fellfield, crowberry mire, earth base of huge boulder; AT123, AT221, AT18-39, AT28-36, AT88-651, AT88-653, AT88-1069, AT104-34, 115241, 115340, 115287A, 115359, 115732 (mixed with Nardia geoscyphus, Barbilophozia sudetica) (BS \& JV), 115804 (BS), 115809 (mixed with Barbilophozia sudetica) (BS), 116058 (BS), 116138, 116198 (BS), 116357 (mixed with Diplophyllum albicans) (BS); reported by Clark \& Frye (1948), Frye \& Clark (1946); Beach Road, Casco Cove, Coast Artillery Hill, Henderson River, Jarmin Pass, Jim Fish Valley, Peaceful Valley, Robinson Ridge, Temnac Cove, Terrible Mountain, Thirty-Seven Hill, West Mountains; Bering Island (Bakalin 2005); IIIc.

Neoorthocaulis floerkei (F. Weber \& D. Mohr) L.

Söderstr., De Roo \& Hedd. [Barbilophozia floerkei (Web. \& Mohr) Loeske]

Common; wet edge of pond, bank of mire slope, alpine slope, wet late snowbed area, alpine blueberry heath, building foundation; mixed with Barbilophozia sudetica, crowberry tundra, dry outcrop shelf; AT16-29, 115248 (mixed with Solenostoma sp.)(BS), 115390 (BS), 115600 (BS), 115613A (BS), 115630 (BS), 115729, 115733 (BS), 115886A (BS), 115908, 116150, 116184 (mixed with Barbilophozia sudentica) (BS), 116254 (BS), 116258 (BS), 120154, 120342, 120359, 120372; reported by Clark \& Frye (1948); Jarmin Pass, Peaceful Valley, Robinson Ridge, Terrible Mountain, USCG Loran C Station; Bering Island (Bakalin 2005) as Orthocaulis floerkei; IIIb.

\section{Pellia endiviifolia (Dicks.) Dumort.}

Rare; shaded bank; 115538; reported by Clark \& Frye (1948), Frye \& Clark (1946); Thirty-Seven Hill; Vb.

\section{Pellia neesiana (Gottsche) Limpr.}

Uncommon; bottom of humus hole, shaded cliff shelves, shaded bank; 115367, 116137, 116148, 120201; reported by Clark \& Frye (1948), Frye \& Clark (1946); Henderson River, Lake Elwood, Robinson Ridge, Terrible Mountain; Bering Island (Bakalin 2005); IVa.

\section{Plagiochila asplenioides (L.) Dumort.}

Reported by Davison (1993) as Plagiochila arctica from Clark \& Frye (1949), Persson (1968); Ic.

* Plagiochila porelloides (Torr. ex Nees) Lindenb. [P. asplenioides ssp. porelloides (Torr. ex Nees) Kaal.]

Rare; up base of cliff in outcrop on slope, humus bank of lake shore, deeply shaded crevices of seaside outcrop; 115272, 120484, 120532; Casco Cove, Siddens Valley, West Mountains; Bering Island (Bakalin 2005); Va.

\section{Plagiochila schofieldiana Inoue}

Uncommon; overhanging sod in seep streamlet, wall of hole in gully, shaded cliff ledge, walls of damp hole in gully, bank of herb shaded gully; 115321, 115513, 115694, 115903, 116045A, 116187, 116238 (mixed with Blepharostoma trichophyllum, Diplophyllum taxifolium, Diplophyllum albicans, Scapania sp., Acrobolbus ciliatus), 120214; 120358; reported by 
Schuster (1980); Gilbert Ridge, Jarmin Pass, Peaceful Valley, Thirty-Seven Hill, West Mountains; VIb. [Endemic to Pacific Northwest North America].

\section{Porella cordaeana (Huebener) Moore}

Uncommon; shaded herb gully, shaded cliff shelf; 115789 , 120423; reported by Persson (1952); Beach Road, Terrible Mountain; IIIg.

Porella fauriei (Steph.) S. Hatt. [P. vernicosa Lindb. subsp. fauriei (Steph.) M. Hara

Uncommon; dune turf on cliff shelf, floor of steep herb slope, cliff shelf base; AT23-36, 115419, 115700, 120448; reported by Hattori (1970); Alexai Point, Gilbert Ridge; VIc.

*Preissia quadrata (Scop.) Nees.

Rare; shaded cliff; 115867; Alexai Point; Bering Island (Bakalin 2005); IVa.

\section{Ptilidium ciliare (L.) Hampe}

Uncommon; crowberry heath, herb slope, old timber from derelict building; AT6-19, 115612, 116250, 120341; reported by Frye \& Clark (1946); Terrible Mountain, Peaceful Valley, Henderson River, West Mountains; Bering Island (Bakalin 2005); IVa (bipolar).

\section{*Ptilidium pulcherrimum (Weber) Vain.}

Rare; old timber from derelict building; 116104 (mixed with Lophozia wenzelii); Peaceful Valley; Va.

\section{Radula complanata (L.) Dumort.}

Uncommon; rock outcrop; AT88-694, AT694, AT704 (BS), 115466, 115774, 116229, 120444, 120449; reported by Clark \& Frye (1948), Frye \& Clark (1946); Alexai Point, Gilbert Ridge, Nevidiskov Creek, Terrible Mountain; Bering Island (Bakalin 2005, 2010a); Va.

Radula obtusiloba Steph. [R. obtusiloba subsp. polyclada (A. Evans) S. Hatt., R. polyclada A. Evans]

Rare; shaded cliff shelf, rock outcrop, damp cliff crevices; 410, 469, 115508, 115539, 115550, 115957 (BS), 115961, $116209,116278,120241,120352,120494$; reported by Clark \& Frye (1948), Frye \& Clark (1946), Persson (1947); Beach Road, Cape Wrangell, Casco Point, Gilbert Ridge, Thirty-Seven Hill, Nevidiskov Creek, Terrible Mountain, West Mountains; Ve.

\section{* Radula prolifera Arnell}

Uncommon; damp overhang along streamlet seepage, damp shaded base of cliff, walls of herb gully, deeply shaded gully; 115329, 115938, 115703, 115792, 120346; Beach Road, Gilbert Ridge, Kingfisher Creek, Terrible Mountain, West Mountains; Copper Island (Bakalin 2010a). Id.

\section{Riccardia chamedryfolia (With.) Grolle}

Rare; damp bank of streamlet; 116267; reported by Davison (1993) from a collection of Sharp 9081 (TENN); Terrible Mountain; Va.

\section{Riccardia multifida (L.) Gray}

Rare; shaded damp bank by waterfall, damp bank of streamlet; 115368 (BS), 116266; reported by Clark \& Frye (1948), Persson (1952); Terrible Mountain; Va (bipolar).

\section{Scapania curta (Mart.) Dumort.}

Rare; shaded bank of river; 116359 (BS); reported by Clark \& Frye (1948); Henderson River; Bering Island Bakalin 2005); IVa.

*Scapania degenii Schiffn. ex Müll. Frib. [Scapania brevicaulis Taylor]

Rare; cliff shelf; 115421 (BS \& JV); USGS Loran C Station; IIa.

\section{Scapania irrigua (Nees) Nees}

Rare; Lyngbye sedge wet meadow, AT41-20 (mixed with Gymnocolea inflata)(BS \& JV), AT42-28 (JV; mixed with Cephalozia bicuspidata), AT43-29 (mixed with Cephalozia bicuspidata, Trilophozia quinquedentata)(BS); reported by Clark \& Frye (1948); O’Donnell Valley, West Massacre Valley; Bering Island (Bakalin 2005); IVa

\section{Scapania paludosa (Müll. Frib.) Müll. Frib.}

Abundant; seepage slope, among sedges at pond margin, seepage, shaded wet hole in gully, wet cliff, damp area, shaded wet floor of humus hole in gully, edge of old runway, seepage slope, wet late snowbed area; 88-606 (PD), AT6013 (BS), AT61-27, 115298A (BS),115400A (BS), 115593A (BS), 115695 (mixed with Dichodontium pellucidum) (BS), 115749 (BS), 116010 (BS), 116032 (mixed with Diplophyllum albicans, Blepharostoma trichophyllum) (BS), 116046 (BS), 116089 (BS), 116149 (BS); reported by Persson (1968); Alexai Point, Beach Road, Chirikof Point, Coast Artillery Hill, Kingfisher Creek, Lake Elwood, Peaceful Valley, Robinson Ridge, USCG Loran C Station; Bering Island (Bakalin 2005); IIIb.

* Scapania parvifolia Warnst. [S. scandica (Arnell \& H. Buch) Macvicar form parvifolia (Warnst.) Schljakov]

Rare; cliff ledge; 115552 (BS); Casco Point; Bering Island (Bakalin 2005); VIII

\section{Scapania scandica (Arnell \& H. Buch) Macvicar}

Uncommon; cliff shelf, damp area by road, wet sedge meadow; AT39-36 (BS), AT39-37 (mixed with Cephalozia bicuspidata), 115933 (BS), 115552 (BS) 115982 (BS); reported by Davison (1993) from a collection of Sharp 9136 (TENN); Beach Road, O’Donnell Valley, USCG Loran C Station; IIb.

\section{Scapania subalpina (Nees ex Lindenb.) Dumort.}

Rare; peaty stream bank, roadside ditch bank, alluvial valley, wet cliff; AT88-800, 115591 (mixed with Calypogeia muelleriana)(BS), 115754 (OL), 115885 (mixed with Hygrobiella laxifolia)(BS), 116077 (JV); reported by Davison (1993) from a collection of Sharp 9323 (TENN); Coast Artillery Hill, O’Donnell Valley; Bering Island (Bakalin 2005); IVa.

\section{Scapania uliginosa (Lindenb.) Dumort.}

Uncommon; water of streamlet, seep area near streamlet, shaded wet depression, damp area, floating in spring pool margin, Lyngbye sedge wet meadow, wet depression in mesic mire, wet depression of seepy slope, wetland margin, seepage area of tufted bulrush slope; AT41-24, AT42-27, AT43$25,115320,115333,115397,115595 \mathrm{~A}, 115635,115662$, 115794, 115652 (mixed with Marsupella emarginata), 115653 (mixed with Marsupella emarginata)(BS), 115834 (BS), 116016 (BS), 116049 (mixed with Scapania paludosa)(BS), 116214 (BS), 120252, 120256, 120288, 120297, 120516, 120168; reported by Macoun (1902); Coast Artillery Hill, Jarmin Pass, Kingfisher Creek, Lake Elwood, Peaceful Valley, Terrible Mountain, Thirty-Seven Hill, USCG Loran C Station; IIIb.

\section{Scapania undulata (L.) Dumort.}

Abundant; damp slope, seepage near road, deep humus hole in tundra, rocks in subalpine streamlet, black masses in wet area, seep in rock by waterfall, boulder in stream, Lyngbye sedge wet meadow, boulder in stream; AT601, 115266 (BS), 115338A (mixed with Jungermannia borealis, Blindia acuta) (BS), 115389A (BS), 115686 (BS), 115806 (mixed with Marsupella emarginata)(BS), 115869 (BS), 116052 (mixed with Calliergon sp.) (BS), 116322 (BS); reported by Clark \& Frye (1948, 1949), Frye \& Clark (1946); Alexai Point, Chirikof Point, Coast Artillery Hill, Murder Point, Peaceful Valley, Terrible Mountain, USCG Loran C Station, West Massacre Valley; Bering Island (Bakalin 2005); IIIa. 
*Schistochilopsis grandiretis (Lindb. ex Kaal.) Konstant. [Lophozia grandiretis (Lindb. in Kaal.) Schiffn.] Rare; wet bank above stream, damp bank of streamlet; 115361 (BS \& JV), 116268 (mixed with Scapania uliginosa, Riccardia multifida, Calypogeia cf. sphagnicola) (JV); Terrible Mountain; IIa.

Schistochilopsis incisa (Schrad.) Konstat. [Lophoẓia incisa (Schrad.) Dumort.]

Uncommon; late snow bed area, sheltered area at base of outcrop, shaded outcrop, damp cliff on humus; 115735 (BS), 115736 (mixed with Barbilophozia sudetica)(BS), 116143; reported by Davison (1993); Henderson River, Kingfisher Creek, Robinson Ridge; Bering Island (Bakalin 2005); IVa.

Schistochilopsis opacifolia (Culm. ex Meyl.) Konstant. [Lophozia opacifolia Culm. ex Meyl., L. incisa subsp opacifolia (Culm. ex Meyl.) R.M. Schust. et Damsh.]

Rare; wet late snow area; 116143; reported by Davison (1993) as L. incisa subsp. opacifolia; Robinson Ridge; Bering Island (Bakalin 2005); IIa.

*Schljakovianthus quadrilobus (Lindb.) Konstant. et Vilnet [Barbilophozia quadriloba (Lindb.) Loeske, Lophozia quadriloba (Lindb.) A. Evans]

Rare; flatlands near house foundation; 116090 (BS); Pleasant Valley. IIb.

Solenostoma fusiforme (Steph.) R.M. Schust. [Jungermannia fusiformis (Steph.) Steph.]

Rare; wet outcrop; reported by Váňa (1975); IIh.

Solenostoma obovatum (Nees) C. Massal. [Jungermannia obovata Nees]

Rare; seep in rock crevice by waterfall, damp bank by stream, rock outcrop; AT604 (BS \& JV, mixed with Marsupella emarginata), AT691 (mixed with Cephalozia bicuspidata), 116060, 116065 (JV), 116069 (JV), 116070 (JV), 116118 (BS), 116280 (BS \& JV, mixed with Cephalozia bicuspidata, Marsupella emarginata); reported by Clark \& Frye $(1948,1949)$, Frye \& Clark (1946); Chirikof Point, Henderson River, Peaceful Valley, Terrible Mountain, West Arm Nevidiskov Bay; Bering Island (Bakalin 2005 as Plectocolea obovata); IIa.

*Solenostoma rubrum (Gottsche) R.M. Schust. [Jungermannia rubra Gottsche, Nardia rubra (Gottsche) A. Evans] Rare; 115589 (JV), 116350 (JV); peaty stream bank, damp bank of river; Coast Artillery Hill, Henderson River; IIIe.

Solenostoma sphaerocarpum (Hook.) Steph. [Jungermannia sphaerocarpa Hook.]

Reported by Clark \& Frye (1948); IVa (bipolar).

Sphenolobus minutus (Schreb. ex D. Crantz) Berggr. [Anastrophyllum minutum (Schreb) R.M. Schuster]

Common; damp outcrop, alpine blueberry heath, bank of humus hole in gully, cliff crevice, pendant humus over outcrop, crowberry heath, humus on cliff shelf, on mound at edge of seepage slope, bluejoint-alpine blueberry heath; AT36-42, AT 37-43, 115546 (BS), 115692, 115854, 116344, 120442; reported by Persson (1952); Alexai Point, Casco Point, Coast Artillery Hill, Gilbert Ridge, Henderson River, Terrible Mountain; Bering Island (Bakalin 2005); IVa (bipolar).

Sphenolobus saxicola (Schrad.) Steph. [Anastrophyllum saxicola (Schrad.) R.M. Schuster]

Common; crowberry and alpine blueberry heaths, tufted clubrush mesic mire; AT 6-42, AT 36-41, AT37-36, AT 22-41; reported by Persson (1952); Gilbert Ridge, Terrible Mountain; IVa.

Trilophozia quinquedentata (Huds.) Bakalin [Tritomaria quinquedentata (Huds.) H. Buch]
Common; alpine blueberry heath, shaded bank, sedge wetland, ditch near road, wall of humus hole, streamlet bank, side of herb shaded gully, fellfield; 115615 (BS), 116001 (BS), 116241 (mixed with Racomitrium lanuginosum), 116256 (mixed with Fuscocephaloziopsis pleniceps)(BS); reported by Clark \& Frye (1948); Coast Artillery Hill, Peaceful Valley, Lake Elwood, Terrible Mountain, West Arm Nevidiskov Bay; Bering Island (Bakalin 2005); IVa.

\section{Analysis of the flora}

The distribution patterns (Konstantinova 2000) of 112 Attu liverworts species indicate three geographic elements dominate the flora: 1) arctomontane (30 species, or $26.1 \%$ ), 2) arctoboreomontane (27 species, or $23.4 \%$ ), and 3) montane $(23$ species, $20 \%$ ). These are followed by a boreal element which includes a moderate number of species $(16$ species, or $13.9 \%$ ), a smaller temperate component (7 species or $6.1 \%$ ), and a nearly absent arctic element (2 species, or $1 \%$ ) (Table 1). In the arctomontane, arctoboreomontane, and boreal elements, many of the species are circumpolar, or nearly circumpolar, and account for approximately $78 \%$ of the flora.

Comparison of the major geographical elements for the North Holarctic (Konstantinova 2000) with those of Attu Island is given in Table 2. With the exception of the higher percent of the Arctic species in the North Holarctic region (16\%), geographic patterns exhibited for Attu Island are similar. This is consistent with the interpretation of Tuhkanen (1984), that Attu Island is boreal and not Arctic. In the central Aleutian Islands, Talbot et al. (2010) reported a higher percentage of boreal plants, $25 \%$, than arctic-alpine, $12 \%$, and a mixture of arctic and boreal classes, $6 \%$. Additionally, Bakalin (2010b) indicated that the Kuril Island Archipelago extends $1200 \mathrm{~km}$ in a northeast direction from Hokkaido Island, Japan, uniting the boreal flora of southern Kamchatka with the temperate flora enriched with subtropical elements of Hokkaido.

\section{Commonness of species}

Table 3 indicates the most important liverwort species within the landscape in terms of commonness. Abundant species included Anthelia julacea, A. juratzkana, Conocephalum salebrosum, Gymnocolea inflata, Herbertus dicranus, Marsupella emarginata, Nardia scalaris, Scapania paludosa, while common species were Barbulophozia sudetica, Blepharostoma trichophyllum, Calopogeia muelleriana, Cephalozia bicuspidata, Jungermannia exsertifolia subsp. cordifolia, Lophozia longiflora, Mylia talorii, Neorthocaulis floerkei and Sphenolobus minutus.

\section{Comparison with the Commander Islands}

A total of 112 liverwort species are reported from the Commander Islands (Bakalin 2010a). Of these species, 43 liverworts, or $38 \%$, have not been reported from Attu Island (Table 4): Athalamia byalina, Barbilophozia hatcheri, Calypogeia integristipula, Cephaloziella elachista, C. uncinata, Conocephalum japonicum, Eremonotus myriocarpus, Fuscocephaloziopsis leucantha, F. pachycaulis, Jungermannia exsertifolia subsp. exsertifolia, J. polaris, Lophocolea heterophylla, L. minor, Lophozia savicziae, L. schusterana, L. silvicola, L. silvicoloides, Lophoziopsis pellucida, Marsupella sprucei, Mesoptychia gillmanii, M. heterocolpos, Mylia 
Table 1. Percent distribution of Attu Island, Alaska, liverwort species in phytogeographic categories following the North Holarctic categories of Konstantinova (2000).

\begin{tabular}{|c|c|c|}
\hline Phytogeographic category & $\begin{array}{l}\text { Number } \\
\text { of species }\end{array}$ & $\begin{array}{l}\text { Percent of } \\
\quad \text { flora }\end{array}$ \\
\hline $\begin{array}{l}\text { I. ARCTIC } \\
\text { d. Siberian-American }\end{array}$ & 2 & 1.8 \\
\hline $\begin{array}{l}\text { II. ARCTOMONTANE } \\
\text { a. Circumpolar } \\
\text { b. Subcircumpolar } \\
\text { d. Disjunctive } \\
\text { e. Euro-Siberian } \\
\text { h. Eurasian-West American } \\
\text { k. Siberian-West American }\end{array}$ & $\begin{array}{l}16 \\
3 \\
6 \\
1 \\
3 \\
1\end{array}$ & $\begin{array}{l}14.3 \\
2.6 \\
5.4 \\
0.9 \\
2.6 \\
0.8\end{array}$ \\
\hline $\begin{array}{l}\text { III. MONTANE } \\
\text { a. Circumpolar } \\
\text { b. Subcircumpolar } \\
\text { c. Amphioceanic } \\
\text { e. Pacific } \\
\text { f. Disjunctive } \\
\text { g. Euro-West American }\end{array}$ & $\begin{array}{l}4 \\
8 \\
3 \\
4 \\
2 \\
1\end{array}$ & $\begin{array}{l}3.6 \\
7.1 \\
2.6 \\
3.6 \\
1.8 \\
0.9\end{array}$ \\
\hline $\begin{array}{l}\text { IV. ARCTOBOREOMONTANE } \\
\text { a. Circumpolar } \\
\text { b. Subcircumpolar } \\
\text { c. Amphioceanic }\end{array}$ & $\begin{array}{c}25 \\
1 \\
1\end{array}$ & $\begin{array}{l}22.3 \\
0.9 \\
0.9\end{array}$ \\
\hline $\begin{array}{l}\text { V. BOREAL } \\
\text { a. Circumpolar } \\
\text { b. Subcircumpolar } \\
\text { e. Euro-American }\end{array}$ & $\begin{array}{l}12 \\
3 \\
1\end{array}$ & $\begin{array}{l}10.7 \\
2.6 \\
0.9\end{array}$ \\
\hline $\begin{array}{l}\text { VI. TEMPERATE } \\
\text { b. Amphioceanic } \\
\text { c. Pacific }\end{array}$ & $\begin{array}{l}6 \\
1\end{array}$ & $\begin{array}{l}5.3 \\
0.9\end{array}$ \\
\hline VII. COSMOPOLITAN & 4 & 3.6 \\
\hline VIII. UNCLEAR & 4 & 3.6 \\
\hline TOTAL & 112 & 100 \\
\hline
\end{tabular}

anomala, Nardia compressa, Neoorthocaulis binsteadii, Obtusifolium obtusum, Odontoschisma elongatum, Plagiochila arctica, Porella platyphylla, Ptilidium californicum, Riccardia aeruginosa, R. latifrons, Saccobasis polita, Sautera alpina, Scapania cuspiduligera, S. byperborea, S. kaurinii, S. ligulata, S. mucronata, S. obscura, Schljakovia kunzeana, Solenostoma gracillimum, S. byalinum, S. infuscum and S. obovatum (Bakalin 2010a). The number of Attu liverworts present in the Commander Islands was 71 , or $63 \%$.

A total of 112 liverwort species were reported from Attu Island, of these 48 liverwort species, or $41 \%$ have not been reported from the Commander Islands (Table 4): Acrobolbus ciliatus, Anastrepta orcadensis, Barbilophozia barbata, Bazzania tricrenata, B. trilobata, Calypogeia azurea, Diplophyllum obtusifolium, Frullania nisqualensis, Gymnomitrion commutatum, G. obtusum, Gyrothyra underwoodiana, Herbertus delavayi, $H$. dicranus, H. sendtneri, Kurzia pauciflora, Lepidozia reptans, Lophozia murmanica, L. guttulata, Marsupella aquatica, M. sphacelata, Metacalypogeia cordifolia, Metzgeria conjugata, M. pubescens, Moerckia bibernica, Mylia talorii, Pellia endiviifolia, Plagiochila asplenioides, P. schofieldiana, Porella cordaeana, P. fauriei, Ptilidium pulcherrimum, Radula obtusiloba, Riccardia chamedryfolia, R. multifida, Scapania degenii, S. uliginosa, Schistochilopsis grandirentis, S. opacifolia, Solenostoma fusiforme, and S. rubrum. These data indicated the liverworts of the region were generally widespread species, but there are large gaps in species distributions between the eastern and western portions of the region.
Table 2. Percent distribution of liverwort species in major North Holarctic elements (Konstantinova 2000) compared to Attu Island, Alaska (present study).

\begin{tabular}{lcc}
\hline Geographic element & North Holarctic & Attu Island \\
\hline Arctic & 16 & 2 \\
Arctomontane & 24 & 27 \\
Montane & 16 & 20 \\
Arctoboreomontane & 19 & 24 \\
Boreal & 15 & 14 \\
Temperate & 10 & 6 \\
Cosmopolitan & 1 & 4 \\
Total & $\mathbf{1 0 0}$ & $\mathbf{1 0 0}$
\end{tabular}

Table 3. Frequency of occurrence of liverwort species in four commonness classes within Attu Island, Alaska.

\begin{tabular}{lcc}
$\begin{array}{l}\text { Commonness } \\
\text { category }\end{array}$ & $\begin{array}{c}\text { Number of } \\
\text { species }\end{array}$ & Percent of flora \\
Rare & 54 & 48.2 \\
Uncommon & 24 & 21.4 \\
Common & 11 & 9.8 \\
Abundant & 9 & 8.1 \\
No data* & 14 & 12.5 \\
\hline Total & $\mathbf{1 1 2}$ & $\mathbf{1 0 0}$ \\
\hline
\end{tabular}

* Published without commonness data.

\section{Liverworts excluded from Attu Island list}

Two unconfirmed Lejeunea species were reported by Davison (1993:224) in a collection of A.J. Sharp 9039 and 9039c (TENN) but no firm determination was made: Lejeunea cf. flava and L. cf. stevensiana growing in admixture with Acrobolbus ciliatus. We searched for these two species at what appeared to be the same and similar sites but did not locate them. Their presence is therefore in doubt, and they may have been incorrectly named. From a geographical perspective, L. flava is a Pantropical species with incursions into the temperate zone, and L. stevensiana is a Himalayan species not occurring outside the Himalayas. We excluded both species from the flora until a definite determination may be made.

\section{DISCUSSION}

This research represented an essential contribution to the understanding of liverwort distribution in a strategic location that bridges the flora between Asia and North America. Our list of Attu liverworts was based on modern liverwort taxonomy which was established through a collaboration of taxonomic specialists (Söderström et al. 2015, 2016). The listed species often reference earlier names from the literature as an aid for the reader.

Our list of liverworts collected from Attu Island updates assessments of liverwort diversity on Attu Island - these results confirm the presence of many previously reported species, add new site records, and add a number of new species records for Attu Island. These include the addition of 34 species to the liverwort flora of Attu Island and markedly increases the total number of species from ca 84 reported by Davison (1993) to a total of 112 species. This $30 \%$ increase suggested previous knowledge of Attu liverwort diversity was incomplete and the liverwort flora is richer than previously known. For logistical reasons, our 
Table 4. Comparison of the numbers of liverwort species present on Attu Island, Alaska, and the Commander Islands, Russian Federation (with percentages based on the total liverwort flora).

\begin{tabular}{|l|l|l|}
\hline Region & Attu Island & Commander Islands \\
\hline Total number of species & 112 & 112 \\
\hline Number of Attu species & present on Commander Islands - 66 (59\%) & absent on Commander Islands - 48 (41\%) \\
\hline Number of Commander species & absent on Attu Island - 41 (37\%) & present on Attu Island - 68 (59\%) \\
\hline
\end{tabular}

study concentrated primarily on the easternmost portions of Attu Island; future field studies from a wider area are anticipated to yield new records. One species, Herbertus delavayi was new to the Aleutian Islands and Alaska; its nearest known reported record is from Haida Gwaii, previously known as the Queen Charlotte Islands, British Columbia (Heinrichs et al. 2010).

The Commander Islands and Attu Island are geographically separated by $300 \mathrm{~km}$. For the vascular flora, Tatewaki (1963:194) pointed out a distinct difference between the Commander Islands and the U.S. Aleutian Islands and proposed the existence of a 'floristic depression' between the two districts. Our results, combined with the work of Bakalin \& Cherdantseva (2008), support the concept of floristic depression in liverwort distribution observed in vascular plants. The significance of Hultenia as a link between the liverwort floras of Asia and America, and the Tatewaki-line in dividing the Aleutian Islands from the Commander Islands is of great value in liverwort bryogeography (Bakalin \& Cherdanseva 2008). For example, Hultenia may have served as a bridge between North America and Asia harboring relicts of Cold and Warm Pleistocene epochs (Bakalin \& Cherdanseva 2008).

Our study provided newly reported species and further distributional evidence. For a number of species, the Aleutians are outposts of their western or eastern extremities of distribution. However, while Hultenia may play a significant role as a migration path for liverworts (Bakalin \& Cherdantseva 2008), it is not possible to indicate the exact number of species - some Aleutian arc taxa may be isolated and separated from their main distribution as relics of warmer epochs. Regardless, our list of Attu Island liverwort species provided a baseline to interpret future floristic change from factors such as climate and ash deposition from volcanic eruption on the liverwort flora.

\section{ACKNOWLEDGEMENTS}

Funding was provided by the Alaska Region, U.S. Fish \& Wildlife Service. We are grateful to John G. Brewer for map production. We are indebted to the U.S. Coast Guard for providing air transportation to and quarters on Attu Island. We thank the captain and crew of the R/V Tiglax for safe passage in the western Aleutian Islands. John M. Pearce, John W. Martin, Hunter A. Gravley, Barbara Ralston, and two anonymous referees provided reviews of prior drafts. Any use of trade, firm, or product names is for descriptive purposes only and does not imply endorsement by the U.S. Government. The findings and conclusions in this article are those of the author(s) and do not necessarily represent the views of the U.S. Fish and Wildlife Service.

\section{LITERATURE CITED}

Anderson, S.T. \& T.P. Bank II. 1952. Pollen and radiocarbon studies of Aleutian soil profiles. Science 116:84-86.

Bakalin, V.A. 2005. The liverwort flora of Bering Island (North-West Pacific, Russia). Lindbergia 30:79-92.

Bakalin, V.A. 2009. Hepatics Marchantiophyta, Anthocerotophyta) flora and phytogeography of Kamchatka and adjacent islands. KMK Scientific Press, Moscow (in Russian). ББакамин В.А. 2009. Фцора и фитогеография печеночников (Marchantiophyta, Anthocerotophyta) Камчатки и прилегающих островов. Москва: Товарищество научных изданий КМК. 367 с.].

Bakalin, V.A. 2010a. The distribution of bryophytes in the Russian Far East. Part 1. Hepatics. Far Eastern University Press, Vladivostok, 175 pp. (in Russian). [Бакалин В.A. 2010. Распространение мохообразных на российском АаАьнем Востоке. Часть 1. Печеночники. ВАадивосток: ИзА-во АВГУ. 175 с.].

Bakalin, V.A. 2010b. Hepaticae of the Kuril Islands (northwestern Pacific): a transoceanic route from Circumboreal to East Asian flora. Annales Botanici Fennici 47:81-105.

Bakalin, V.A. \& V.Ya. Cherdantseva 2008. Bryophyte flora of Medny Island and bryogeography of the Aleutians (North Pacific). In: Conservation of biodiversity of Kamchatka and coastal waters. Proceedings of VIII International Scientific Conference, Petropavlovsk-Kamchatsky, November 27-28, 2007, V.F. Bugaev, A.M. Tokranov \& O.A. Chernyagina (eds), pp. 36-56. Kamchatpress, Petropavlovsk-Kamchatsky. [Бакамин В.А., Черданцева В.Я. 2008. Бриофлора острова Медный и биогеография Алеут (Северная Пацифика) // Сохранение биоразнообразия Камчатки и прилегающих морей: Аоклады VIII межАународной научной конференции, посвященной 275-летию с начала Второй Камчатской экспедиции (1732-1733 гг.). Петропавловск-Камчатский: Камчатпресс. С. 36-56].

Bank, T.P. II. 1951. Aleutian vegetation and Aleut culture. Michigan Academy of Arts \& Letters Papers 37:13-30.

Clark, L. \& T.C. Frye 1948. Attu Island Hepaticae collected by Howard. The Bryologist 51:252-255.

Clark, L. \& T.C. Frye 1949. A small collection of Alaskan Hepaticae. The Bryologist 52:58-61.

Damsholt, K. 2002. Illustrated flora of Nordic liverworts and hornworts. Nordic Bryological Society, Lund, 840 pp.

Davison, P.G. 1993. Floristic and phytogeographic studies of the hepatic flora of the Aleutian Islands, Alaska. Ph.D., University of Tennessee, Knoxville, TN, 385 pp.

Davison, P.G. \& D.K. Smith 1992. Calycularia crispula (Hepaticae) in the Aleutian Islands and Pacific Northwest of North America. The Bryologist 95:266-269.

Elias, S.A. \& B. Crocker 2008. The Bering Land Bridge: a moisture barrier to the dispersal of steppe-tundra biota? Quaternary Science Reviews 27:2473-2483.

Evans, A.W. 1900. Notes on the Hepaticae collected in Alaska. Papers from the Harriman Alaska Expedition V. Proceedings of the Washington Academy of Sciences II: 287-314. 
Farr, W.A. \& J.S. Hard 1987. Multivariate analysis of climate along the southern coast of Alaska - some forestry implications. Research Paper PNW-RP-372. U.S. Department of Agriculture, Forest Service, Pacific Northwest Research Station, Portland, Oregon, 38 pp.

Feldberg, K., J. Váňa, J. Krusche, J. Kretschmann, S.D.F. Patzak, O.A. Pérez-Escobar, N.R. Rudolf, N. Seefelder, A. Schäfer-Verwimp, D.G. Long, H. Schneider \& J. Heinrichs 2016. A phylogeny of Cephaloziaceae (Jungermanniopsida) based on nuclear and chloroplast DNA markers. Organisms Diversity \& Evolution 16(4):727-742.

Frye, T.C. \& L. Clark 1946. Attu Island bryophytes collected by Hardy. The Bryologist 49:59-65.

Gallant, A.L., E.F. Binnian, J.M. Omernik \& M.B. Shasby 1995. Ecoregions of Alaska. U.S. Geological Survey Professional Paper 1567. Washington, D.C., 73 pp. + map.

Gates, O., H.A. Powers \& R.E. Wilcox 1971. Geology of the Near Islands, Alaska with a section on Surficial geology of the Near Islands by John P. Schafer. Geological Survey Bulletin 1028-U. U. S. Printing Office, Washington, D.C., 822 pp. +3 plates.

Harter, D.E.V., D.H.I. Severin, S. Bumsuk, M.J. Steinbauer, R. Gillespie, K.A. Triantis, J-M. Fernández-Palaciao \& C. Beierkuhnlein. 2015. Impacts of global climate change on the floras of oceanic island - Projections, implications and current knowledge. Perspectives in Plant Ecology, Evolution and Systematics 17:160-183.

Hattori, S. 1970. Studies of the Asiatic species of the genus Porella (Hepaticae). III. Journal of the Hattori Botanical Laboratory 33:41-87.

Heinrichs, J., K. Feldberg, H-P. Kreier \& J. Váňa, 2010. DNAbased identification of Herbertus species on Gough Island, South Atlantic Ocean. Cryptogamie Bryologie 31:67-74.

Heusser, C.J. 1990. Late Quaternary vegetation of the Aleutian Islands, southwestern Alaska. Canadian Journal of Botany 68:1320-1326.

Hickman, J.C. (ed.) 1993. The Jepson manual: higher plants of California. University of California Press, Berkeley, California, $1400 \mathrm{pp}$.

Hong, W.S. 1980. A study of the distribution of Diplophyllum in western North America. The Bryologist 83:497-504.

Hultén, E. 1960. Flora of the Aleutian Islands and westernmost Alaska Peninsula with notes on the flora of the Commander Islands. 2nd edition. Hafner, New York, NY, 376 pp.

Hultén, E. 1968. Flora of Alaska and neighboring territories. Stanford University Press, Stanford, California, 1008 pp.

Ivanov, A.N. 2003. Landscape's features of the Commander Islands. Izvestiya Russkogo Geograficheskogo Obshchestva 135(1):64-70 (in Russian). [Иванов А.Н. 2003. АанАшафтные особенности Командорских островов // Известия Русского географического общества. Т. 135, № 1. C. 64-70].

Jongmans, A.G., N. van Breemen, S.R. Gradstein \& F. van Oort 2001. How liverworts build hanging gardens from volcanic ash in Costa Rica. Catena 44:13-22.

Juslén, A. 2006. Revision of Asian Herbertus (Herbertaceae, Marchantiophyta). Annales Botanici Fennici 43: 409-436.

Konstantinova, N.A. 2000. Distribution patterns of the North Holarctic hepatics. Arctoa 9: 29-94.

Konstantinova, N.A. \& Yu.S. Mamontov 2010. A revision of the genus Calycularia Mitt. (Calyculariaceae, Marchantiophyta). Arctoa 19:117-130.

Kowal, J., S. Pressel, J.G. Duckett \& M.I. Bidartondo. 2016. Liverworts to the rescue: An investigation of their efficacy as mycorrhizal inoculum for vascular plants. Functional Ecology 30:1014-1023.
Krestov, P.V. 2004. Plant cover of the Commander Islands. Botanicheskii Zhurnal 89:1740-1762 (in Russian). [Крестов П.В. 2004. Растительный покров Командорских островов // Ботанический журнал. Т. 89, № 11. C. 1740-1762].

Krestov, P.V., A.M. Omelko \& Y. Nakamura 2010. Phytogeography of higher units of forests and krummholz in North Asia and formation of vegetation complex in the Holocene. Phytocoenologia 40:41-56.

Leslie, L.D. 1989. Alaska climate summaries: a compilation of longterm means and extremes at 478 Alasken stations. 2nd ed. Anchorage, Alaska: Arctic Environmental Information and Data Center, University of Alaska Anchorage, 478 pp.

Lieth, H., J. Berlekamp, S. Fuest \& S. Riediger 1999. Climate diagram World atlas, CD-Series I of Climate and Biosphere, 1st ed. Backhuys Publishers, Leiden, The Netherlands.

Macoun, J. 1902. Lichens and Hepaticae. Catalog of Canadian plants, Part VII. Geological Survey of Canada, Government Printing Bureau, Ottawa, Canada, 318 pp.

Patric, J.H. \& P.E. Black 1968. Potential evapotranspiration and climate in Alaska by Thornthwaite's classification. U.S. Department of Agriculture, Forest Service Research Paper PNW-71. Pacific Northwest Forest \& Range Experiment Station, Portland, Oregon, 28 pp.

Persson, H. 1946. Some Alaskan and Yukon bryophytes. The Bryologist 49:41-58.

Persson, H. 1947. Further notes on Alaskan-Yukon bryophytes. The Bryologist 50:279-310.

Persson, H. 1952. Critical or otherwise interesting bryophytes from Alaska-Yukon. The Bryologist 55:1-25, 88-116.

Persson, H. 1968. Bryophytes from the Aleutian Islands, collected mainly by Hansford T. Shacklette. Svensk. Botanisk. Tidskrift 62:369-387.

Schofield, W.B. 2002. Field guide to liverwort genera of Pacific North America. University of Washington Press, Seattle, 228 pp.

Schuster, R.M. 1980. The Hepaticae and Anthocerotae of North America east of the bundredth meridian. Vol. IV. Columbia University Press, New York, 1334 pp.

Sharp, A.J. \& S. Hattori 1968. Acrobolbus ciliatus from Attu. Journal of Japanese Botany 43:311-315.

Smith, N.R. \& R.F. Griggs 1932. The microflora of the ash of Katmai Volcano with especial reference to nitrogen fixing bacteria. Soil Science 34:365-374.

Söderström, L., A. Hagborg \& M. von Konrat 2015. Liverworts from the largest of the United States: a checklist for mainland Alaska. Arctoa 24:327-361.

Söderström, L., A. Hagborg, M. von Konrat, S. Bartholomew-Began, D. Bell, L. Briscoe, E. Brown, D.C. Cargill, D.P. Costa, B.J. Crandall-Stotler, E.D. Cooper, G. Dauphin, J.J. Engel, K. Feldberg, D. Glenny, S.R. Gradstein, X. He, J. Heinrichs, J. Hentschel, A.L. Ilkiu-Borges, T. Katagiri, N.A. Konstantinova, J. Larraín, D.G. Long, M. Nebel, T. Pócs, F. Puche, E. Reiner-Drehwald, M.A.M. Renner, A. Sass-Gyarmati, A. Schäfer-Verwimp, J.G. Segarra Moragues, R.E. Stotler, P. Sukkharak, B.M. Thiers, J. Uribe, J. Váňa, J. Carlos Villarreal, M. Wigginton, L. Zhang \& R.-L. Zhu. 2016. World checklist of hornworts and liverworts. PhytoKeys 59:1-828.

Talbot, S.S. \& S.L. Talbot 1994. Numerical classification of the coastal vegetation of Attu Island, Aleutian Islands, Alaska. Journal of Vegetation Science 5:867-876.

Talbot, S.S., W.B. Schofield, S.L. Talbot \& F.J.A. Daniëls 2010. Vegetation of eastern Unalaska Island, Aleutian Islands, Alaska. Botany 88:366-388.

Talbot, S.S., S.L. Talbot \& L.R. Walker 2010. Post-eruption legacy effects and their implications for long-term reco- 
very of the vegetation on Kasatochi Island, Alaska. Arctic, Antarctic, and Alpine Research 42:285-296.

Tatewaki, M. 1963. Hultenia. Journal of the Faculty of Agriculture, Hokkaido University, S3 53: 131-199.

Tatewaki, M. \& Y. Kobayashi 1934. A contribution to the flora of the Aleutian Islands. Journal of the Faculty of Agriculture of the Hokkaido Imperial University 36:1-119.

Thornthwaite, C.W. 1948. An approach toward a rational classification of climate. Geographical Review 38:55-94.

Tsvetkov, A.A., A.V. Fedorchuk \& A.Yu. Gladenkov 2010. Geology and magmatic evolution of Bering Island. International Geology Review 32(12):1202-1217.
Tuhkanen, S. 1984. A circumboreal system of climatic-phytogeographical regions. Acta Botanica Fennica 127:1-50 + appendices.

Váňa, J. 1975. Miscellaneous notes on the Asiatic Jungermannioideae IV. Journal of the Hattori Botanical Laboratory 39:211-214.

Vilnet, A.A., N.A. Konstantinova \& A.V. Troitsky 2012. Molecular phylogeny and systematics of the suborder Cephaloziineae with special attention to the family Cephaloziaceae s.l. (Jungermanniales, Marchantiophyta). Arctoa 21:113-132. 